\title{
When rare meets common: Treatable genetic diseases are enriched in the general psychiatric population
}

\author{
Venuja Sriretnakumar ${ }^{1,2}$, Ricardo Harripaul ${ }^{1,3}$, James L. Kennedy ${ }^{1,4}$, \\ Joyce So ${ }^{1,2,4,5,6}$
}

${ }^{1}$ Campbell Family Mental Health Research Institute, Centre for Addiction and Mental Health, Toronto, Canada

${ }^{2}$ Department of Laboratory Medicine and Pathobiology, University of Toronto, Toronto, Canada

${ }^{3}$ Institute of Medical Science, University of Toronto, Toronto, Canada

${ }^{4}$ Department of Psychiatry, University of Toronto, Toronto, Canada

${ }^{5}$ Department of Medicine, University of Toronto, Toronto, Canada

${ }^{6}$ Division of Medical Genetics, Departments of Medicine and Pediatrics, University of California, San Francisco, USA

KEY WORDS: inborn errors of metabolism; channelopathies; schizophrenia; bipolar disorder; major depressive disorder; general anxiety disorder; obsessive compulsive disorder; psychiatric genetics

To be published 


\subsection{Summary}

Mental illnesses are one of the biggest contributors to the global disease burden. Despite the increased recognition, diagnosis and ongoing research of mental health disorders, the etiology and underlying molecular mechanisms of these disorders are yet to be fully elucidated. Moreover, despite many treatment options available, a large subset of the psychiatric patient population is nonresponsive to standard medications and therapies. There has not been a comprehensive study to date examining the burden and impact of treatable genetic disorders (TGDs) that can present with neuropsychiatric features in psychiatric patient populations. In this study, we test the hypothesis that TGDs that present with psychiatric symptoms are more prevalent within psychiatric patient populations compared to the general population by performing targeted next-generation sequencing (NGS) of 129 genes associated with 108 TGDs in a cohort of 2301 psychiatric patients. In total, 72 putative affected and 293 putative carriers for TGDs were identified, with known or likely pathogenic variants in 78 genes. Despite screening for only 108 genetic disorders, this study showed an approximately four-fold (4.13\%) enrichment for genetic disorders within the psychiatric population relative to the estimated $1 \%$ cumulative prevalence of all single gene disorders globally. This strongly suggests that the prevalence of these, and most likely all, genetic diseases are greatly underestimated in psychiatric populations. Increasing awareness and ensuring accurate diagnosis of TGDs will open new avenues to targeted treatment for a subset of psychiatric patients.

\subsection{Introduction}

Mental illnesses are one of the biggest contributors to the global disease burden, with major depressive disorder (MDD) alone being the second highest contributor (Vigo et al., 2016). Despite the increased recognition, diagnosis and 
ongoing research of mental health disorders, the etiology and underlying molecular mechanisms of these disorders are yet to be fully elucidated. Moreover, despite many treatment options available, a large subset of the psychiatric patient population are non-responsive to standard medications and therapies (Bokma et al., 2019; Fabbri et al., 2019; Middleton et al., 2019; Mizuno et al., 2020; Sanches et al., 2019).

Decades of research has established that all of the common psychiatric illnesses - MDD, generalized anxiety disorder (GAD), schizophrenia spectrum disorders (SSD), bipolar disorder (BPD) and obsessive-compulsive disorder (OCD) - have contributing genetic factors (Gordovez et al., 2020; Liu et al., 2019; Meier et al., 2019; Ormel et al., 2019; Purty et al., 2019). However, there is a paucity of research and literature regarding the contribution of rare, highly penetrant genetic variants underlying psychiatric disorders. The limited studies have primarily focused on specific inborn errors of metabolism (IEMs), a subgroup of inherited genetic disorders wherein defects in proteins or enzymes along metabolic pathways result in toxic accumulations of substrates or metabolites (Bauer et al., 2013; Olivier Bonnot et al., 2019; Demily et al., 2017; Simons et al., 2017; Sriretnakumar et al., 2019; Trakadis et al., 2018), and chromosomal copy number variants (Charney et al., 2019; Cleynen et al., 2020; Rees et al., 2014; Sriretnakumar et al., 2019; Tansey et al., 2016). IEMs have been of particular interest due to the availability of targeted therapies and treatments for many of these disorders. (Saudubray et al., 2018; Waters et al., 2018). Although newborn screening and early presentation has led to many IEMs being primarily diagnosed in infancy and early childhood, many IEMs are now known to have late-onset presentations that are more prevalent than previously thought (Saudubray et al., 2009). Significantly, many late-onset IEMs present with psychiatric manifestations that can be indistinguishable from primary psychiatric disorders (Sedel et al., 2007; Sriretnakumar et al., 2019; Trakadis et al., 2018), and there have been 
numerous case reports of patients with late-onset IEMs who have been misdiagnosed as having a primary mental illness (Simons et al., 2017; Walterfang et al., 2013). Furthermore, heterozygote carriers for some autosomal recessive IEMs have been reported to present with psychiatric symptoms that can be ameliorated by treating the underlying genetic condition (Cocco et al., 2009; Tarnacka et al., 2009). Besides IEMs, there are other TGDs that can present with psychiatric phenotypes, including triplet repeat expansion disorders, neurocutaneous disorders and channelopathies (Cabal-Herrera et al., 2020; Kleopa, 2011; Northrup et al., 2018; Peng et al., 2018; Ratna et al., 2020). Examples of treatments for genetic disorders include specific drugs, such as miglustat for the treatment of Niemann-Pick disease type C (Patterson et al., 2020) and copper chelators for Wilson disease (Litwin et al., 2019), lifestyle modifications, such as avoidance of alcohol and fasting for acute intermittent porphyria (Fontanellas et al., 2016), dietary treatment, such as low-protein diet in urea cycle defects or phenylketonuria (Häberle et al., 2019), vitamin supplementation, such as folic acid, and vitamins B6 and B12 for homocystinuria (Jitpimolmard et al., 2020), and antiepileptic drugs for channelopathies, amongst many others (Baraban et al., 2013; Knupp et al., 2018; Pastor et al., 2018; Wolff et al., 2019). There is also some preliminary evidence of a role for variants associated with rare genetic disorders being associated with treatment nonresponsiveness in psychiatric patients (Sriretnakumar et al., 2019), though further comprehensive study is needed.

There has not been a comprehensive study to date examining the burden and impact of TGDs in psychiatric populations. In this study, we test the hypothesis that TGDs that present with psychiatric symptoms are more prevalent within psychiatric patient populations compared to the general population by performing targeted next-generation sequencing (NGS) of 129 genes associated with 108 TGDs in a cohort of 2301 psychiatric patients. 


\subsection{Materials and Methods}

\subsubsection{Samples}

A total of 2301 DNA samples from psychiatric patients were analyzed in this study. The patient cohort is a sub-sample retrieved from a larger sample set collected as part of the Individualized Medicine: Pharmacogenetic Assessment \& Clinical Treatment (IMPACT) study at the Centre for Addiction and Mental Health (CAMH; Toronto, Canada). Sample characteristics are described elsewhere (Herbert et al., 2018; IMPACT, 2017). The demographic characteristics and psychiatric diagnoses of the current study cohort are provided in Table 4.1. Patient consent for genetic testing was obtained at the time of study recruitment, and research ethics board approval for this study was obtained through $\mathrm{CAMH}$ (Toronto, Canada).

\subsubsection{DNA Sequencing}

NGS was performed for 129 genes associated with 108 TGDs that have been associated with psychiatric phenotypes (supplementary Table S4.1). Probes for targeted sequencing of the 129 genes were designed on the Agilent Technologies SureDesign online platform (https://earray.chem.agilent.com/suredesign/). DNA samples were purified using Agencourt AMPure XP (Beckman Coulter Life Sciences, Indianapolis, IN) and quantified using Qubit ${ }^{\mathrm{TM}}$ dsDNA BR Assay Kit (ThermoFisher Scientific Inc., Waltham, MA). Agilent Technologies (Santa Clara, CA) SureSelectXT protocol for $3 \mu \mathrm{g}$ of input DNA was followed for library preparation, hybridization, and capture. NGS was carried out on the NovaSeq SP flow cell (300 cycles) (Illumina Inc., San Diego, CA). 
Table 4.1 Demographic characteristics of the study sample $(n=2301)$.

\begin{tabular}{|c|c|c|c|c|c|c|c|}
\hline & SSD* & BPD $^{* *}$ & $O C D^{* * *}$ & $\begin{array}{l}\text { MDD } \\
\text { only }\end{array}$ & $\begin{array}{l}\text { GAD } \\
\text { only }\end{array}$ & $\begin{array}{l}\text { MDD- } \\
\text { GAD }\end{array}$ & Total \\
\hline $\begin{array}{l}\text { Number of } \\
\text { Sample }\end{array}$ & 436 & 556 & 375 & 179 & 302 & 453 & 2301 \\
\hline $\begin{array}{c}\text { Male : Female } \\
\%\end{array}$ & $\begin{array}{l}308: 128 \\
71: 29\end{array}$ & $\begin{array}{c}177: 379 \\
32: 68\end{array}$ & $\begin{array}{c}161: 214 \\
43: 57\end{array}$ & $\begin{array}{l}46: 133 \\
26: 74\end{array}$ & $\begin{array}{c}105: 197 \\
35: 65\end{array}$ & $\begin{array}{c}123: 330 \\
27: 73\end{array}$ & $\begin{array}{c}920: 1 \\
381 \\
40: 60\end{array}$ \\
\hline \multicolumn{8}{|l|}{ Ethnicity (\%) } \\
\hline African & $40(9)$ & $6(1)$ & $4(1)$ & $3(1)$ & $2(1)$ & $5(1)$ & $60(3)$ \\
\hline Caucasian & $298(69)$ & $481(86)$ & $320(85)$ & $147(82)$ & $260(86)$ & $403(89)$ & $\begin{array}{c}1909 \\
(83)\end{array}$ \\
\hline East Asian & $18(4)$ & $12(2)$ & $8(2)$ & $8(5)$ & 7 (2) & $10(2)$ & $63(3)$ \\
\hline Hispanic & $1(0)$ & $5(1)$ & $1(0)$ & $4(2)$ & $3(1)$ & $4(1)$ & $18(1)$ \\
\hline $\begin{array}{l}\text { Mixed } \\
\text { Ethnicity }\end{array}$ & $22(5)$ & $13(2)$ & $13(4)$ & $7(4)$ & $12(4)$ & $11(2)$ & 78 (3) \\
\hline $\begin{array}{l}\text { Not } \\
\text { Available }\end{array}$ & $31(7)$ & $33(6)$ & $26(7)$ & $10(6)$ & $14(4)$ & 15 (3) & $129(6)$ \\
\hline $\begin{array}{l}\text { Indigenous } \\
\text { People } \\
\text { (Canada) }\end{array}$ & $5(1)$ & $3(1)$ & $0(0)$ & $0(0)$ & $2(1)$ & $2(1)$ & $12(0)$ \\
\hline $\begin{array}{l}\text { South } \\
\text { Asian }\end{array}$ & $21(5)$ & $3(1)$ & $3(1)$ & $0(0)$ & $2(1)$ & $3(1)$ & $32(1)$ \\
\hline
\end{tabular}

*83 patients had a secondary psychiatric diagnosis. ${ }^{*} 203$ patients had a secondary psychiatric diagnosis. ${ }^{* *} 28$ patients had a secondary psychiatric diagnosis. SSD, schizophrenia spectrum disorders; BPD, bipolar disorder; MDD, major depressive disorder; $O C D$, obsessive compulsive disorder; GAD, generalized anxiety disorder. 


\subsubsection{Bioinformatic Analyses}

Sequence alignment, variant calling, and variant annotation were performed using in-house scripts on the CAMH Specialized Computing Cluster (see supplementary methods for more information). Variant classification was carried out based on American College of Medical Genetics guidelines (Richards et al., 2015) through the use of the Human Genetic Mutation Database $\left(\mathrm{HGMD}^{\circledR}\right)$ Professional 2020.1 (Stenson et al., 2017), ClinVar (Landrum et al., 2018) and Franklin by Genoox (https://franklin.genoox.com/clinical-db/home) platforms. Additional manual literature search was also conducted when discrepancies between the three platforms arose. See supplementary Table S4.3 for citations of all known pathogenic variants identified in this study sample.

\subsubsection{Protein Modelling}

Protein modelling was performed for likely pathogenic (LP) variants and variants of uncertain significance (VUSes) for disease genes where protein models were available from the Protein Data Bank (see supplementary Table S4.1 for further information). Protein modelling was carried out on PyMOL Molecular Graphics System Version 2.4 (Schrödinger LLC, New York City, NY). The most likely rotamer was chosen to depict the amino acid change of non-synonymous variants.

\subsubsection{Statistical Analysis}

Exact binomial test was used for statistical comparison of observed pathogenic variants versus expected variant frequencies. Expected variant frequencies were derived from established disease prevalence for autosomal dominant disorders and males affected with X-linked disorders, where available (supplementary Table S4.2). Expected carrier frequencies for autosomal recessive disorders and rates of females heterozygous for $\mathrm{X}$-linked disorders were 
calculated based on disease prevalence and assuming Hardy-Weinberg equilibrium (HWE) when specific carrier rates were not available (supplementary Table S4.2). T-test was used to compare the mean number of medications in patients with and without variants of interest (i.e. LP or pathogenic variants). All statistical tests were performed using R version 3.6.0 (Team, 2017), all tests were 2-sided and all p-values were Bonferroni-corrected.

\subsection{Results}

\subsubsection{Variants of Interest}

Figure 4.1 summarizes the breakdown of all sequenced variants following bioinformatic analysis. All exons sequenced averaged 300X read depth per sample. A total of 1748 variants were identified following annotation and filtration. Of these, a total of 207 pathogenic and 215 LP variants were identified for further statistical comparison. All pathogenic and LP variants identified in the study population were either absent or found at very low frequencies in ethnicity-matched Genome Aggregation Database (gnomAD v2:

http://gnomad.broadinstitute.org/) exome sub-populations (supplementary Table S4.3).

Table 4.2 shows the breakdown of patients identified with genetic variants of interest sorted by psychiatric diagnosis. Pathogenic or LP variants were identified in a total of 100 SSD, 64 BPD, 98 OCD, 22 MDD, 32 GAD and 49 MDDGAD patients, with the highest frequency of pathogenic and LP variants identified in the OCD (39\%) and SSD (28.2\%) patient subsets.

There were also 22 patients identified to carry multiple variants within the same recessive disease-associated gene. Patient 6544 with OCD was identified to have two LP variants within the $A S L$ gene. All other identified variants within this 
group were classified to be VUSes and thus were excluded from further statistical analysis.

\subsubsection{Protein Modelling}

Protein models were available to perform modelling of 40 LP variants and 29 VUSes (supplementary Tables S4.3 and S4.4). Of this, 33 LP missense variants resulted in changes to amino acid interactions within the protein, while seven LP variants did not result in any changes. However, two of the seven variants without protein interaction changes have previously known pathogenic variants at the exact same location and the remaining five variants are located in the same region as previously known pathogenic variants. For VUSes, 25 out of the 29 variants modeled resulted in significant amino acid interaction changes within the protein. Of the four variants that did not result in any protein changes, two variants are located in the same region as previously established pathogenic variants. 


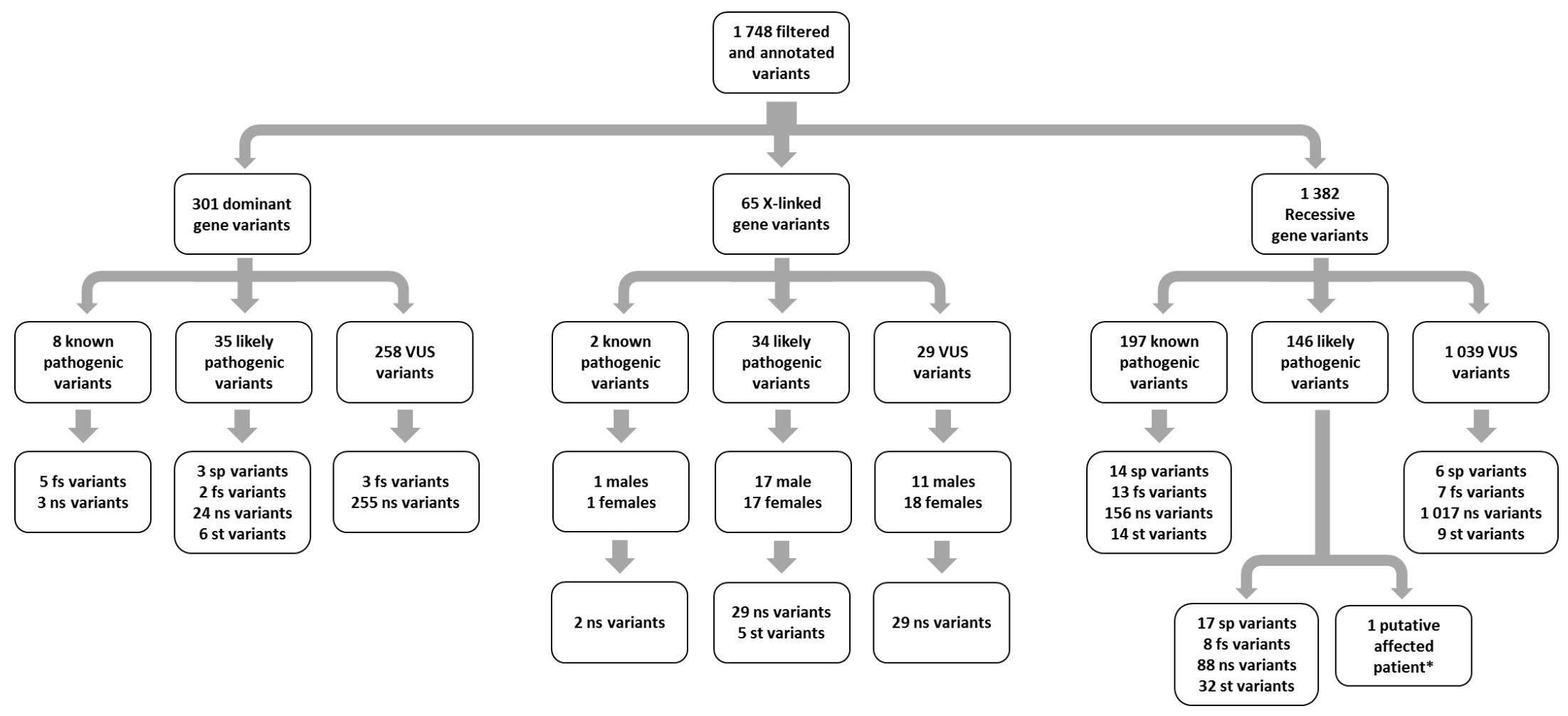

Figure 4.1 Diagram depicting the filtered and annotated variant breakdown in all 2301 SSD, BPD, OCD, MDD only, GAD only, and MDD-GAD samples. ${ }^{*}$ One patient with 2 likely pathogenic variants in the same recessive gene. VUS, variant of uncertain significance; fs, frameshift; ns, nonsynonymous; sp, splice; st, stop gain/stop loss. 
Table 4.2 Number of patients with genetic variants of interest identified by psychiatric diagnosis.

\begin{tabular}{|c|c|c|c|c|c|c|c|}
\hline DISORDER TYPE & SSD & BPD & OCD & $\begin{array}{c}\text { MDD } \\
\text { Only }\end{array}$ & $\begin{array}{c}\text { GAD } \\
\text { Only }\end{array}$ & $\begin{array}{c}\text { MDD- } \\
\text { GAD }\end{array}$ & $\begin{array}{c}\text { Total Patients } \\
\text { by Disorder } \\
\text { Type }\end{array}$ \\
\hline $\begin{array}{c}\text { Autosomal } \\
\text { Dominant } \\
\text { (putative } \\
\text { affected) }\end{array}$ & $14^{* * *}$ & 6 & $12^{* * *}$ & 3 & 2 & 6 & 43 \\
\hline $\begin{array}{c}\text { X-linked } \\
\text { (putative } \\
\text { affected)* }\end{array}$ & 5 & 9 & 8 & 2 & 2 & 3 & 29 \\
\hline $\begin{array}{c}\text { X-linked } \\
\text { (putative } \\
\text { carriers) ** }\end{array}$ & 0 & 2 & 0 & 0 & 0 & 0 & 291 \\
\hline $\begin{array}{c}\text { Autosomal } \\
\text { Recessive } \\
\text { (putative carriers) }\end{array}$ & 81 & 47 & 78 & 17 & 28 & 40 & \\
\hline $\begin{array}{c}\text { Total Patients by } \\
\text { Diagnosis }\end{array}$ & 100 & 64 & 98 & 22 & 32 & 49 & 365 \\
\hline
\end{tabular}

*Denotes all males with $\mathrm{X}$-linked disorder variants, and females with presenting $X$-linked disorder variants. ** Denotes all females with non-presenting $X$-linked disorder variants. *** Denotes two patients putative affected for two different genetic disorders (found to have variants associated with both an autosomal dominant disorder and presenting X-linked disorder). SSD, schizophrenia spectrum disorders; BPD, bipolar disorder; MDD, major depressive disorder; OCD, obsessive compulsive disorder; GAD, generalized anxiety disorder.

\subsubsection{Prevalence of Treatable Genetic Disorder Variants within the Study Cohort}

Patients with pathogenic or LP variants in genes associated with autosomal dominant disorders and male patients with pathogenic or LP variants in genes associated with X-linked disorder genes were considered to be putatively affected with the disorder. Those heterozygous for a pathogenic or LP 
variant in an autosomal recessive disorder gene and female patients heterozygous for a pathogenic or LP variant in an X-linked disorder gene were considered to be carriers for the disorder. The observed numbers of psychiatric patients who are putatively affected with and/or carriers for TGDs in comparison to expected disease prevalences and carrier frequencies are summarized in Table 4.3. The prevalences of autosomal dominant disorders related to pathogenic variants in CACNA1A (episodic ataxia type 2, familial hemiplegic migraine type 1, spinocerebellar ataxia type 6), SCN1A (epilepsy/febrile seizures, Dravet syndrome, familial hemiplegic migraine type 3), SCN2A (early infantile epileptic encephalopathy and/or benign familial infantile seizures), SCN3A (early infantile epileptic encephalopathy and/or benign familial infantile seizures), COQ2 (susceptibility to multiple system atrophy), HMBS (acute intermittent porphyria; AIP), PPOX (porphyria variegate), TTR (hereditary amyloidosis), NAGLU (autosomal dominant Charcot-Marie-Tooth disease type 2V), and GCH1 (doparesponsive dystonia), and $X$-linked disorders related to pathogenic variants in $A B C D 1$ (X-linked adrenoleukodystrophy (X-ALD) and/or adrenomyeloneuropathy), OTC (ornithine transcarbamylase (OTC) deficiency), PDHA1 (pyruvate dehydrogenase E1-alpha (PDHA1) deficiency) and SLC6A8 (cerebral creatine deficiency syndrome 1) were significantly higher in the study cohort than expected based on general population prevalence, with the exception of OTC deficiency in females. There was an increased carrier rate for variants of interest in COQ9 (biallelic mutations in which are associated with primary coenzyme Q10 (CoQ10) deficiency-5) and GCDH (biallelic mutations in which are associated with glutaric academia type 1 (GA1)) within the OCD subset of the study cohort relative to the general population. 
Table 4.3 Prevalence of observed treatable IEM pathogenic variant frequencies in the psychiatric population compared to expected disease prevalence/carrier rates in the general population.

\begin{tabular}{|c|c|c|c|c|c|c|c|c|c|}
\hline \multirow[b]{2}{*}{ Gene } & \multirow[b]{2}{*}{ Disease/Phenotype } & & \multicolumn{7}{|c|}{ Psychiatric Disorders } \\
\hline & & $\begin{array}{l}\text { Prevalencel } \\
\text { Carrier Rate* }\end{array}$ & $\begin{array}{l}\operatorname{SSD}(n=436) \\
(95 \% \mathrm{Cl}, \mathrm{p}- \\
\text { value })\end{array}$ & $\begin{array}{l}\text { BPD }(n=556) \\
(95 \% \mathrm{Cl}, \mathrm{p}- \\
\text { value })\end{array}$ & $\begin{array}{c}\text { MDD }(n=179) \\
(95 \% \mathrm{Cl}, \mathrm{p}- \\
\text { value })\end{array}$ & $\begin{array}{l}O C D(n=375) \\
(95 \% \mathrm{Cl}, \mathrm{p}- \\
\text { value })\end{array}$ & $\begin{array}{l}\mathrm{GAD}(\mathrm{n}=302) \\
(95 \% \mathrm{Cl}, \mathrm{p}- \\
\text { value })\end{array}$ & $\begin{array}{l}\text { MDD-GAD } \\
(n=453)(95 \% \\
\text { Cl, p-value) }\end{array}$ & $\begin{array}{c}\text { Total } \\
(\mathrm{n}=2301) \\
(95 \% \mathrm{Cl}, \mathrm{p}- \\
\text { value })\end{array}$ \\
\hline \multicolumn{10}{|c|}{ Dominant Genes } \\
\hline \multirow{3}{*}{ CACNA1A } & Early infantile epileptic encephalopathy & unknown & 5 & 5 & 0 & 6 & 0 & 4 & 20 \\
\hline & $\begin{array}{c}\text { Episodic ataxia type } 2 \text { and/or familial hemiplegic } \\
\text { migraine with progressive cerebellar ataxia } \\
\text { and/or spinocerebellar ataxia }\end{array}$ & $1 / 100000$ & $\begin{array}{l}5(0.0037- \\
0.0266 \\
6.39 \mathrm{E}-14)\end{array}$ & $\begin{array}{l}5(0.0026- \\
0.0209 \\
2.16 \mathrm{E}-13)\end{array}$ & 0 & \begin{tabular}{|}
$6(0.0059-$ \\
$0.0345,1.1 \mathrm{E}-$ \\
$15)$
\end{tabular} & 0 & $\begin{array}{l}4(0.0024- \\
0.0224 \\
8.63 \mathrm{E}-11)\end{array}$ & $\begin{array}{c}20(0.0053- \\
0.0134,1.1 \mathrm{E}- \\
15)\end{array}$ \\
\hline & Familial hemiplegic migraine & $3 / 100000$ & $\begin{array}{l}5(0.0037- \\
0.0266 \\
1.54 \mathrm{E}-11)\end{array}$ & $\begin{array}{l}5(0.0029- \\
0.0209 \\
5.21 \mathrm{E}-11)\end{array}$ & 0 & $\begin{array}{l}6(0.0059- \\
0.0345 \\
1.34 \mathrm{E}-14)\end{array}$ & 0 & $\begin{array}{l}4(0.0024- \\
0.0224 \\
6.94 \mathrm{E}-09)\end{array}$ & $\begin{array}{c}20(0.0053- \\
0.0134,1.1 \mathrm{E}- \\
15)\end{array}$ \\
\hline \multirow[t]{2}{*}{ COQ2 } & Susceptibility to multiple system atrophy & $4.9 / 100000$ & $\begin{array}{l}1 \text { (5.82E-05- } \\
1.27 \mathrm{E}-02 \\
0.1057)\end{array}$ & 0 & 0 & 0 & $\begin{array}{l}1(8.38 \mathrm{E}-05- \\
1.83 \mathrm{E}-02 \\
\mathbf{0 . 0 4 4 )}\end{array}$ & 0 & $\begin{array}{l}2(0.0001- \\
0.0031 \\
0.0177)\end{array}$ \\
\hline & Primary coenzyme Q10 deficiency & unknown & 1 & 0 & 0 & 0 & 1 & 0 & 2 \\
\hline HMBS & Acute intermittent porphyria & $1 / 100000$ & 0 & 0 & $\begin{array}{c}1(0.0001- \\
0.0307 \\
\mathbf{0 . 0 0 3 5 8 )}\end{array}$ & 0 & 0 & 0 & $\begin{array}{c}1(0.0000- \\
0.0024 \\
\mathbf{0 . 0 4 5 5 )}\end{array}$ \\
\hline$P P O X$ & Porphyria variegata & $3.2 / 1000000$ & 0 & 0 & $\begin{array}{l}1(0.0001- \\
0.0307 \\
\mathbf{0 . 0 0 1 2})\end{array}$ & 0 & 0 & 0 & $\begin{array}{l}1(0.0000- \\
0.0024 \\
0.0147)\end{array}$ \\
\hline SCN1A & Generalized epilepsy with febrile seizures & unknown & 6 & 1 & 1 & 4 & 1 & 2 & 15 \\
\hline
\end{tabular}




\begin{tabular}{|c|c|c|c|c|c|c|c|c|c|}
\hline & $\begin{array}{l}\text { Early infantile epileptic encephalopathy (Dravet } \\
\text { syndrome) }\end{array}$ & $1 / 15700$ & $\begin{array}{c}6(0.0051- \\
0.0297 \\
4.21 \mathrm{E}-12)\end{array}$ & \begin{tabular}{|c}
1 (4.56E-05- \\
$9.98 \mathrm{E}-03$ \\
$0.209)$
\end{tabular} & $\begin{array}{c}1(0.0001- \\
0.0307 \\
0.0794)\end{array}$ & $\begin{array}{c}4(0.0029- \\
0.0271 \\
9.17 \mathrm{E}-08)\end{array}$ & \begin{tabular}{|c}
1 (8.38E-05- \\
$1.83 \mathrm{E}-02$ \\
$\mathbf{0 . 0 1 3 3})$
\end{tabular} & $\begin{array}{c}2(0.0005- \\
0.0159 \\
0.0029)\end{array}$ & $\begin{array}{c}15(0.0037- \\
0.0107 \\
1.54 \mathrm{E}-15)\end{array}$ \\
\hline & Familial hemiplegic migraine & $3 / 100000$ & $\begin{array}{c}6(0.0051- \\
0.0297 \\
4.65 \mathrm{E}-14)\end{array}$ & $\begin{array}{c}1 \text { (4.55E-05- } \\
9.98 \mathrm{E}-03 \\
0.0992)\end{array}$ & $\begin{array}{c}1(0.0001- \\
0.0307 \\
0.0375)\end{array}$ & $\begin{array}{c}4(0.0029- \\
0.0271 \\
4.56 \mathrm{E}-09)\end{array}$ & \begin{tabular}{|c}
1 (8.38E-05- \\
$1.83 \mathrm{E}-02$ \\
$\mathbf{0 . 0 6 3 1})$
\end{tabular} & $\begin{array}{c}2(0.0005- \\
0.0159 \\
6.39 \mathrm{E}-04)\end{array}$ & $\begin{array}{c}15(0.0037- \\
0.0107 \\
1.54 \mathrm{E}-15)\end{array}$ \\
\hline SCN2A & $\begin{array}{l}\text { Early infantile epileptic encephalopathy and/or } \\
\text { benign familial infantile seizures }\end{array}$ & unknown & 1 & 0 & 0 & 1 & 0 & 0 & 2 \\
\hline SCN3A & $\begin{array}{l}\text { Familial focal epilepsy with variable foci and/or } \\
\text { early infantile epileptic encephalopathy }\end{array}$ & unknown & 1 & 0 & 0 & 0 & 0 & 0 & 1 \\
\hline TTR & Transthyretin-related hereditary amyloidosis & $1 / 100000$ & \begin{tabular}{|c}
1 (5.81E-05- \\
$1.27 \mathrm{E}-02$ \\
$\mathbf{0 . 0 0 8 7})$
\end{tabular} & 0 & 0 & 0 & 0 & 0 & $\begin{array}{c}1(0.0000- \\
0.0024 \\
\mathbf{0 . 0 4 5 5 )}\end{array}$ \\
\hline $\mathrm{GCH} 1$ & Autosomal dominant dopa-responsive dystonia & $0.5 / 1000000$ & 0 & $\begin{array}{c}1 \text { (4.55E-05- } \\
9.98 \mathrm{E}-03 \\
\mathbf{0 . 0 0 0 8}\end{array}$ & 0 & 0 & $\begin{array}{c}1 \text { (8.38E-05- } \\
1.83 \mathrm{E}-02 \\
4.53 \mathrm{E}-04)\end{array}$ & 0 & $\begin{array}{c}2(0.0001- \\
0.0031 \\
1.98 \mathrm{E}-06)\end{array}$ \\
\hline NAGLU & $\begin{array}{c}\text { Autosomal dominant Charcot-Marie-Tooth } \\
\text { disease type } 2 \mathrm{~V}\end{array}$ & unknown & 0 & 1 & 0 & 1 & 0 & 1 & 3 \\
\hline \multicolumn{10}{|c|}{ X-linked Genes } \\
\hline $\begin{array}{l}A B C D 1 \\
\text { (Males) }\end{array}$ & $\begin{array}{l}\text { X-linked cerebral adrenoleukodystrophy and/or } \\
\text { Adrenomyeloneuropathy }\end{array}$ & $1 / 200000$ & 0 & $\begin{array}{c}2(0.0004- \\
0.0129 \\
1.54 \mathrm{E}-05)\end{array}$ & 0 & $\begin{array}{c}2(0.0006- \\
0.0191 \\
7.00 \mathrm{E}-06)\end{array}$ & 0 & $\begin{array}{c}1 \text { (5.58E-05- } \\
1.22 \mathrm{E}-02 \\
9.05 \mathrm{E}-03)\end{array}$ & $\begin{array}{c}5(0.0007- \\
0.0051 \\
6.63 \mathrm{E}-12)\end{array}$ \\
\hline $\begin{array}{c}A B C D 1 \\
\text { (Females) }\end{array}$ & $\begin{array}{l}\text { X-linked cerebral adrenoleukodystrophy and/or } \\
\text { Adrenomyeloneuropathy }\end{array}$ & $9.52 \mathrm{E}-06$ & 0 & \begin{tabular}{|c}
1 (4.55E-05- \\
$9.98 \mathrm{E}-04$ \\
$\mathbf{0 . 0 1 5 8})$
\end{tabular} & 0 & $\begin{array}{c}4(0.0029- \\
0.0271 \\
1.99 \mathrm{E}-11)\end{array}$ & 0 & 0 & $\begin{array}{c}5(0.0007- \\
0.0051 \\
1.24 \mathrm{E}-10)\end{array}$ \\
\hline $\begin{array}{c}\text { OTC } \\
\text { (Males) }\end{array}$ & Ornithine transcarbamylase deficiency & $1 / 62000$ & $\begin{array}{c}6(0.0051- \\
0.0297 \\
1.32 E-05)\end{array}$ & $\begin{array}{c}2(0.0004- \\
0.0129 \\
2.39 \mathrm{E}-04)\end{array}$ & 0 & \begin{tabular}{|c}
1 (6.75E-05- \\
$1.47 \mathrm{E}-02$ \\
$\mathbf{0 . 0 3 6 2})$
\end{tabular} & \begin{tabular}{|c}
1 (8.38E-05- \\
$1.83 \mathrm{E}-02$ \\
$\mathbf{0 . 0 2 9 2})$
\end{tabular} & $\begin{array}{c}1 \text { (5.58E-05- } \\
\text { 1.22E-02, } \\
\mathbf{0 . 0 4 3 7 )}\end{array}$ & $\begin{array}{c}11(0.0024- \\
0.0085 \\
1.32 \mathrm{E}-15)\end{array}$ \\
\hline $\begin{array}{c}\text { OTC } \\
\text { (Females) }\end{array}$ & Ornithine transcarbamylase deficiency & 0.008 & 0 & $\begin{array}{l}3(0.0011- \\
0.0157,1)\end{array}$ & $\begin{array}{l}2(0.0014- \\
0.0398,1)\end{array}$ & $\begin{array}{l}1(6.75 \mathrm{E}-05- \\
1.47 \mathrm{E}-02,1)\end{array}$ & 0 & $\begin{array}{l}1(5.58 \mathrm{E}-05- \\
1.22 \mathrm{E}-02,1)\end{array}$ & $\begin{array}{c}7(0.0012- \\
0.0063 \\
0.0230)\end{array}$ \\
\hline $\begin{array}{l}\text { PDHA1 } \\
\text { (Males \& }\end{array}$ & Pyruvate dehydrogenase E1-alpha deficiency & $\begin{array}{l}\sim 200 \text { cases } \\
\text { worldwide }\end{array}$ & 1 & 1 & 0 & 3 & 1 & 0 & 6 \\
\hline
\end{tabular}




\begin{tabular}{|c|c|c|c|c|c|c|c|c|c|}
\hline $\begin{array}{l}\text { Females) } \\
\text { (XLD) }\end{array}$ & & & & & & & & & \\
\hline $\begin{array}{l}\text { SLC6A8 } \\
\text { (Females) }\end{array}$ & Cerebral creatine deficiency syndrome 1 & unknown & 0 & 2 & 0 & 0 & 0 & 0 & 2 \\
\hline \multicolumn{10}{|c|}{ Recessive Genes } \\
\hline$A S L^{* *}$ & Argininosuccinic aciduria & $1 / 70000$ & 0 & 0 & 0 & $\begin{array}{l}1(6.75 \mathrm{E}-05- \\
1.47 \mathrm{E}-02 \\
\mathbf{0 . 0 1 0 7 )}\end{array}$ & 0 & 0 & $\begin{array}{c}1(0.0000- \\
0.0024 \\
0.0647)\end{array}$ \\
\hline ACADM & $\begin{array}{l}\text { Medium chain acyl-CoA dehydrogenase } \\
\text { deficiency }\end{array}$ & $1 / 40$ & $\begin{array}{l}2(0.0006- \\
0.0165 \\
0.0148)\end{array}$ & $\begin{array}{l}3(0.0011- \\
0.0157 \\
0.0047)\end{array}$ & 0 & $\begin{array}{l}1(6.75 \mathrm{E}-05- \\
1.48 \mathrm{E}-02 \\
0.0071)\end{array}$ & 0 & $\begin{array}{c}4(0.0024- \\
0.0224,0.114)\end{array}$ & $\begin{array}{l}10(0.0021- \\
0.008,8.09 \mathrm{E}- \\
14)\end{array}$ \\
\hline$A C A D V L$ & $\begin{array}{l}\text { Very long chain acyl-CoA dehydrogenase } \\
\text { deficiency }\end{array}$ & 0.0115 & $\begin{array}{l}1(5.81 \mathrm{E}-05- \\
1.27 \mathrm{E}-02 \\
0.2828)\end{array}$ & 0 & 0 & $\begin{array}{l}2(0.0006- \\
0.0191 \\
3.86 \mathrm{E}-04)\end{array}$ & $\begin{array}{l}1(8.38 \mathrm{E}-05- \\
1.83 \mathrm{E}-02 \\
0.0501)\end{array}$ & $\begin{array}{l}3(0.0014- \\
0.0192 \\
2.82 \mathrm{E}-06)\end{array}$ & $\begin{array}{l}7(0.0012- \\
0.0063 \\
5.43 \mathrm{E}-05)\end{array}$ \\
\hline ACAT1 & Beta-ketothiolase deficiency & 0.002 & $\begin{array}{c}3(0.0014- \\
0.0199 \\
0.2330)\end{array}$ & 0 & 0 & $\begin{array}{l}1(6.75 \mathrm{E}-05- \\
1.47 \mathrm{E}-02,1)\end{array}$ & 0 & $\begin{array}{l}1(5.58 \mathrm{E}-05- \\
1.22 \mathrm{E}-02,1)\end{array}$ & $\begin{array}{l}5(0.0007- \\
0.0051,1)\end{array}$ \\
\hline$A G A$ & Aspartylglucosaminuria & unknown & 0 & 0 & 0 & 0 & 1 & 0 & 1 \\
\hline$A L D H 3 A 2$ & Sjögren-Larsson syndrome & 0.004 & $\begin{array}{l}1(5.81 \mathrm{E}-05- \\
1.27 \mathrm{E}-02,1)\end{array}$ & 0 & 0 & 0 & 0 & 0 & $\begin{array}{l}1(0.0000- \\
0.0024 \\
0.0047)\end{array}$ \\
\hline ALDH5A1 & $\begin{array}{l}\text { Succinic semialdehyde dehydrogenase } \\
\text { deficiency }\end{array}$ & $\begin{array}{l}\sim 450 \text { cases } \\
\text { worldwide }\end{array}$ & 4 & 0 & 0 & 0 & 0 & 0 & 4 \\
\hline ALDH7A1 & Pyridoxine-dependent epilepsy & $\begin{array}{l}\sim 200 \text { cases } \\
\text { worldwide }\end{array}$ & 1 & 0 & 0 & 0 & 0 & 0 & 1 \\
\hline AMT & $\begin{array}{l}\text { Atypical glycine encephalopathy and/or Infantile } \\
\text { glycine encephalopathy and/or Neonatal glycine } \\
\text { encephalopathy }\end{array}$ & $\begin{array}{l}\sim 20 \text { cases } \\
\text { worldwide }\end{array}$ & 2 & 0 & 1 & 0 & 0 & 0 & 3 \\
\hline APTX & Ataxia-oculomotor apraxia type 1 & unknown & 1 & 0 & 0 & 0 & 0 & 0 & 1 \\
\hline ARSA & $\begin{array}{l}\text { Metachromatic leukodystrophy (late infantile, } \\
\text { juvenile, adult forms) }\end{array}$ & unknown & 5 & 0 & 0 & 2 & 0 & 1 & 8 \\
\hline
\end{tabular}




\begin{tabular}{|c|c|c|c|c|c|c|c|c|c|}
\hline$A S L$ & Argininosuccinic aciduria & 0.0075 & $\begin{array}{l}5(0.0037- \\
0.0266,1)\end{array}$ & $\begin{array}{l}2(0.0004- \\
0.0129,1)\end{array}$ & 0 & $\begin{array}{c}6(0.0059- \\
0.0345,0.327)\end{array}$ & 0 & $\left|\begin{array}{c}6(0.0049- \\
0.0286,0.807)\end{array}\right|$ & $\begin{array}{l}19(0.0050- \\
0.0129,1)\end{array}$ \\
\hline ASS1 & $\begin{array}{c}\text { Acute neonatal citrullinemia type I and/or Adult- } \\
\text { onset citrullinemia type I }\end{array}$ & 0.0095 & $\begin{array}{l}4(0.0021- \\
0.0233,1)\end{array}$ & 0 & 0 & $\begin{array}{l}2(0.0006- \\
0.0191,1)\end{array}$ & 0 & 0 & $\begin{array}{c}6(0.0010- \\
0.0027 \\
4.16 \mathrm{E}-04)\end{array}$ \\
\hline ATP7B & Wilson Disease & $1 / 90$ & $\begin{array}{l}4(0.0025- \\
0.0233,1)\end{array}$ & $\begin{array}{l}6(0.004- \\
0.0233,1)\end{array}$ & $\begin{array}{c}4(0.0061- \\
0.0562,0.979)\end{array}$ & $\begin{array}{l}6(0.0059- \\
0.0345,1)\end{array}$ & $\begin{array}{l}5(0.0054- \\
0.0382,1)\end{array}$ & $\begin{array}{l}2(0.0005- \\
0.0159,1)\end{array}$ & $\begin{array}{l}27(0.0077- \\
0.017,1)\end{array}$ \\
\hline $\begin{array}{c}B C K D H A, \\
B C K D H B, \\
\& D B T\end{array}$ & maple syrup urine disease & 0.0046 & $\mid \begin{array}{c}5(0.0037- \\
0.0265,0.369)\end{array}$ & $\begin{array}{l}3(0.0011- \\
0.0157,1)\end{array}$ & $\begin{array}{l}1(0.0001- \\
0.0307,1)\end{array}$ & $\begin{array}{l}1(6.75 \mathrm{E}-05- \\
1.47 \mathrm{E}-02,1)\end{array}$ & $\begin{array}{c}4(0.0036- \\
0.0335,0.365)\end{array}$ & $\begin{array}{l}4(0.0024- \\
0.0225,1)\end{array}$ & $\begin{array}{c}18(0.0046- \\
0.0123,0.208)\end{array}$ \\
\hline$B T D$ & Biotinidase deficiency & $1 / 120$ & $\begin{array}{c}8(0.0080- \\
0.0358 \\
0.1581)\end{array}$ & 0 & $\begin{array}{l}1(0.0001- \\
0.0307,1)\end{array}$ & 0 & 0 & $\begin{array}{c}1 \text { (5.58E-05- } \\
\text { 1.22E-02, } \\
0.981)\end{array}$ & $\begin{array}{l}10(0.0021- \\
0.008,0.15)\end{array}$ \\
\hline CBS & Homocystinuria & 0.0052 & $\begin{array}{l}2(0.0006- \\
0.0165,1)\end{array}$ & $\begin{array}{l}4(0.0020- \\
0.0183,1)\end{array}$ & 0 & $\begin{array}{l}2(0.0006- \\
0.0191,1)\end{array}$ & 0 & $\begin{array}{l}3(0.0014- \\
0.0192,1)\end{array}$ & $\begin{array}{c}11(0.0024- \\
0.0085 \\
0.0125)\end{array}$ \\
\hline COQ9 & $\begin{array}{l}\text { Encephalopathy-hypertrophic cardiomyopathy- } \\
\text { renal tubular disease syndrome }\end{array}$ & 0.002 & 0 & 0 & 0 & $\begin{array}{c}4(0.0029- \\
0.0271 \\
0.0144)\end{array}$ & 0 & 0 & $\begin{array}{l}4(0.0005- \\
0.0044,1)\end{array}$ \\
\hline$C P$ & Aceruloplasminemia & 0.0014 & $\begin{array}{l}1(5.81 \mathrm{E}-05- \\
1.27 \mathrm{E}-02,1)\end{array}$ & $\begin{array}{l}1(4.55 \mathrm{E}-05- \\
9.98 \mathrm{E}-03,1)\end{array}$ & $\begin{array}{l}1(0.0001- \\
0.0307,1)\end{array}$ & 0 & $\begin{array}{l}1(8.38 \mathrm{E}-05- \\
1.83 \mathrm{E}-02,1)\end{array}$ & $\begin{array}{l}1(5.58 \mathrm{E}-05- \\
1.22 \mathrm{E}-02,1)\end{array}$ & $\begin{array}{l}5(0.0007- \\
0.0051,1)\end{array}$ \\
\hline CPS1 & Carbamoyl-phosphate synthetase 1 deficiency & 0.008 & 0 & 0 & 0 & 0 & 0 & $\begin{array}{l}2(0.0005- \\
0.0159,1)\end{array}$ & $\begin{array}{c}2(0.0001- \\
0.0031 \\
6.59 \mathrm{E}-06)\end{array}$ \\
\hline CUBN & Imerslund-Gräsbeck syndrome & 0.0049 & $\begin{array}{l}1 \text { (5.81E-05- } \\
1.27 \mathrm{E}-02,1)\end{array}$ & 0 & 0 & $\begin{array}{l}2(0.0006- \\
0.0191,1)\end{array}$ & 0 & 0 & $\begin{array}{c}3(0.0003- \\
0.0038 \\
0.0206)\end{array}$ \\
\hline CYP27A1 & Cerebrotendinous xanthomatosis & 0.002 & 0 & $\begin{array}{l}1(4.55 \mathrm{E}-05- \\
9.98 \mathrm{E}-03,1)\end{array}$ & 0 & 0 & 0 & 0 & $\begin{array}{c}1(0.0000- \\
0.0024,0.202)\end{array}$ \\
\hline DHCR7 & Smith-Lemli-Opitz syndrome & $1 / 100$ & $\begin{array}{l}4(0.0025- \\
0.0233,1)\end{array}$ & $\begin{array}{c}1 \text { (4.55E-05- } \\
9.98 \mathrm{E}-03 \\
0.2044)\end{array}$ & 0 & $\begin{array}{l}2(0.0006- \\
0.0191,1)\end{array}$ & 0 & 0 & $\begin{array}{c}7(0.0012- \\
0.0063 \\
8.26 \mathrm{E}--04)\end{array}$ \\
\hline
\end{tabular}




\begin{tabular}{|c|c|c|c|c|c|c|c|c|c|}
\hline$D L A T$ & Pyruvate dehydrogenase E2 deficiency & $\begin{array}{l}4 \text { cases } \\
\text { worldwide }\end{array}$ & 0 & 0 & 0 & 4 & 0 & 3 & 7 \\
\hline $\begin{array}{l}\text { ETFB \& } \\
\text { ETFDH }\end{array}$ & Multiple acyl-CoA dehydrogenase deficiency & 0.006 & $\begin{array}{l}2(0.0006- \\
0.0165,1)\end{array}$ & 0 & 0 & 0 & 0 & 0 & $\begin{array}{c}2 \text { (0.0001- } \\
0.0031 \\
4.19 \mathrm{E}-04)\end{array}$ \\
\hline FOLR1 & $\begin{array}{l}\text { Neurodegenerative syndrome due to cerebral } \\
\text { folate transport deficiency }\end{array}$ & $\begin{array}{l}<20 \text { cases } \\
\text { worldwide }\end{array}$ & 2 & 0 & 0 & 0 & 0 & 0 & 2 \\
\hline GALC & Krabbe disease & $1 / 125$ & $\begin{array}{l}2(0.0006- \\
0.0165,1)\end{array}$ & 0 & $\begin{array}{l}1(0.0001- \\
0.0307,1)\end{array}$ & $\begin{array}{l}3(0.0016- \\
0.0232,1)\end{array}$ & 0 & 0 & $\begin{array}{l}6(0.0010- \\
0.0057 \\
5.77 \mathrm{E}-03)\end{array}$ \\
\hline \multirow{2}{*}{ GBA } & Gaucher disease type 1 & 0.007 & $\begin{array}{l}2(0.0006- \\
0.0165,1)\end{array}$ & 0 & $\begin{array}{l}2(0.0014- \\
0.0398,1)\end{array}$ & $\begin{array}{l}1(6.75 \mathrm{E}-05- \\
1.47 \mathrm{E}-02,1)\end{array}$ & 0 & 0 & $\begin{array}{l}5(0.0007- \\
0.0051 \\
9.78 \mathrm{E}-03)\end{array}$ \\
\hline & $\begin{array}{l}\text { Gaucher disease-ophthalmoplegia- } \\
\text { cardiovascular calcification syndrome and/or } \\
\text { Hereditary late-onset Parkinson disease }\end{array}$ & $\begin{array}{l}<30 \text { cases } \\
\text { worldwide }\end{array}$ & 2 & 0 & 2 & 1 & 0 & 0 & 5 \\
\hline $\mathrm{GCDH}$ & Glutaryl-CoA dehydrogenase deficiency & 0.0061 & 0 & $\begin{array}{l}1(4.55 \mathrm{E}-05- \\
9.98 \mathrm{E}-03 \\
0.8241)\end{array}$ & 0 & $\begin{array}{l}11(0.0147- \\
0.0519 \\
7.68 \mathrm{E}-05)\end{array}$ & 0 & 0 & $\begin{array}{l}12(0.0027- \\
0.0091,1)\end{array}$ \\
\hline $\mathrm{GCH} 1$ & GTP cyclohydrolase I deficiency & 0.002 & 0 & $\begin{array}{l}1(4.55 \mathrm{E}-05- \\
9.98 \mathrm{E}-03,1)\end{array}$ & 0 & 0 & $\begin{array}{l}1(8.38 \mathrm{E}-05- \\
1.83 \mathrm{E}-02,1)\end{array}$ & 0 & $\begin{array}{l}2(0.0001- \\
0.0031,1)\end{array}$ \\
\hline$G L D C$ & $\begin{array}{l}\text { glycine encephalopathy (atypical, infantile, } \\
\text { neonatal forms) }\end{array}$ & 0.004 & $\begin{array}{l}1(5.81 \mathrm{E}-05- \\
1.27 \mathrm{E}-02,1)\end{array}$ & $\begin{array}{l}3(0.0011- \\
0.0157,1)\end{array}$ & 0 & $\begin{array}{l}1(6.75 \mathrm{E}-05- \\
1.48 \mathrm{E}-02,1)\end{array}$ & 0 & 0 & $\begin{array}{c}5(0.0007- \\
0.0051,0.97)\end{array}$ \\
\hline HADHA & $\begin{array}{l}\text { Long chain 3-hydroxyacyl-CoA dehydrogenase } \\
\text { deficiency and/or Mitochondrial trifunctional } \\
\text { protein deficiency }\end{array}$ & unknown & 1 & 1 & 0 & 1 & 0 & 0 & 3 \\
\hline HEXA & Tay-Sachs disease & $1 / 300$ & $\begin{array}{c}3(0.0014- \\
0.020,0.8965)\end{array}$ & $\begin{array}{l}2(0.0004- \\
0.0129,1)\end{array}$ & 0 & $\begin{array}{l}1(6.75 \mathrm{E}-05- \\
1.47 \mathrm{E}-02,1)\end{array}$ & $\begin{array}{l}2(0.0008- \\
0.0237,1)\end{array}$ & 0 & $\begin{array}{l}8(0.0015- \\
0.0111,1)\end{array}$ \\
\hline \multirow{2}{*}{ HGSNAT } & Sanfilippo syndrome type C & 0.0015 & $\begin{array}{l}1(5.81 \mathrm{E}-05- \\
1.27 \mathrm{E}-02,1)\end{array}$ & $\begin{array}{l}1(4.55 \mathrm{E}-05- \\
9.98 \mathrm{E}-03,1)\end{array}$ & 0 & 0 & 0 & 0 & $\begin{array}{l}2(0.0001- \\
0.0031,1)\end{array}$ \\
\hline & Retinitis pigmentosa & 0.0255 & $\begin{array}{l}1 \text { (5.81E-05- } \\
1.27 \mathrm{E}-02 \\
0.001)\end{array}$ & $\begin{array}{l}1 \text { ( } 4.55 \mathrm{E}-05- \\
9.98 \mathrm{E}-03 \\
5.67 \mathrm{E}-05)\end{array}$ & 0 & 0 & 0 & 0 & $\begin{array}{c}2(0.0001- \\
0.0031,6.6 \mathrm{E}- \\
16)\end{array}$ \\
\hline
\end{tabular}




\begin{tabular}{|c|c|c|c|c|c|c|c|c|c|}
\hline HLCS & Holocarboxylase synthetase deficiency & 0.0068 & $\begin{array}{l}2(0.0006- \\
0.0165,1)\end{array}$ & 0 & 0 & 0 & 0 & 0 & $\begin{array}{c}2(0.0001- \\
0.0031 \\
7.46 \mathrm{E}-05)\end{array}$ \\
\hline IDUA & $\begin{array}{l}\text { Hurler syndrome and/or Hurler-Scheie syndrome } \\
\text { and/or Scheie syndrome }\end{array}$ & 0.0063 & $\begin{array}{l}2(0.0006- \\
0.0165,1)\end{array}$ & 0 & 0 & 0 & $\begin{array}{l}1(8.38 \mathrm{E}-05- \\
1.83 \mathrm{E}-02,1)\end{array}$ & 0 & $\begin{array}{c}3(0.0003- \\
0.0038 \\
0.0016)\end{array}$ \\
\hline IVD & Isovaleric acidemia & 0.004 & $\begin{array}{l}1(5.81 \mathrm{E}-05- \\
1.27 \mathrm{E}-02,1)\end{array}$ & $\begin{array}{l}1(4.55 \mathrm{E}-05- \\
9.98 \mathrm{E}-03,1)\end{array}$ & 0 & 0 & 0 & 0 & $\begin{array}{c}2(0.0001- \\
0.0031 \\
0.0352)\end{array}$ \\
\hline MAN2B1 & Alpha-mannosidosis & 0.0028 & $\begin{array}{l}1(5.81 \mathrm{E}-05- \\
1.27 \mathrm{E}-02,1)\end{array}$ & $\begin{array}{l}1(4.55 \mathrm{E}-05- \\
9.98 \mathrm{E}-03,1)\end{array}$ & 0 & $\begin{array}{c}3(0.0017- \\
0.0232,0.358)\end{array}$ & 0 & 0 & $\begin{array}{l}5(0.0007- \\
0.0051,1)\end{array}$ \\
\hline MCCC2 & 3-methylcrotonyl-CoA carboxylase deficiency & 0.0115 & $\begin{array}{c}1 \text { (5.81E-05- } \\
1.27 \mathrm{E}-02 \\
0.4241)\end{array}$ & $\begin{array}{c}2(0.0004- \\
0.0129 \\
0.6342)\end{array}$ & $\begin{array}{l}1(0.0001- \\
0.0307,1)\end{array}$ & $\begin{array}{l}2(0.0006- \\
0.0191,1)\end{array}$ & $\begin{array}{l}2(0.0008- \\
0.0237,1)\end{array}$ & $\begin{array}{l}3(0.0014- \\
0.0192,1)\end{array}$ & $11(0.0081)$ \\
\hline MMAA & $\begin{array}{c}\text { Vitamin B12-responsive methylmalonic acidemia } \\
\text { type cblA }\end{array}$ & unknown & 1 & 0 & 0 & 1 & 0 & 0 & 2 \\
\hline$M M A B$ & $\begin{array}{c}\text { Vitamin B12-responsive methylmalonic acidemia } \\
\text { type cblB }\end{array}$ & unknown & 1 & 1 & 0 & 1 & 0 & 0 & 3 \\
\hline MMACHC & $\begin{array}{l}\text { Methylmalonic acidemia with homocystinuria, } \\
\text { type cblC }\end{array}$ & $\begin{array}{l}\sim 500 \text { cases } \\
\text { worldwide }\end{array}$ & 7 & 3 & 0 & 0 & 0 & 0 & 10 \\
\hline MOCS1 & $\begin{array}{l}\text { Sulfite oxidase deficiency due to molybdenum } \\
\text { cofactor deficiency type A }\end{array}$ & $\begin{array}{l}\sim 100 \text { cases } \\
\text { worldwide }\end{array}$ & 0 & 0 & 0 & 2 & 0 & 0 & 2 \\
\hline $\begin{array}{l}\text { MTR \& } \\
\text { MTRR }\end{array}$ & $\begin{array}{l}\text { Methylcobalamin deficiency type cblG and/or } \\
\text { Methylcobalamin deficiency type cblE }\end{array}$ & $\begin{array}{l}\sim 30 \text { cases } \\
\text { worldwide }\end{array}$ & 1 & 7 & 0 & 0 & 0 & 2 & 10 \\
\hline MTTP & Abetalipoproteinemia & $\begin{array}{l}<100 \text { cases } \\
\text { worldwide }\end{array}$ & 1 & 0 & 0 & 0 & 1 & 0 & 2 \\
\hline MUT & Methylmalonic aciduria & 0.0089 & \begin{tabular}{|c}
1 (5.81E-05- \\
$1.27 \mathrm{E}-02$ \\
$0.987)$
\end{tabular} & $\begin{array}{c}1 \text { (4.55E-05- } \\
9.98 \mathrm{E}-03 \\
0.5275)\end{array}$ & 0 & $\begin{array}{l}1(6.75 \mathrm{E}-05- \\
1.47 \mathrm{E}-02,1)\end{array}$ & $\begin{array}{l}3(0.0021- \\
0.0287,1)\end{array}$ & 0 & $\begin{array}{c}6(0.0010- \\
0.0057 \\
0.0017)\end{array}$ \\
\hline NAGLU & Sanfilippo syndrome type B & 0.0045 & 0 & $\begin{array}{l}1(4.55 \mathrm{E}-05- \\
9.98 \mathrm{E}-03,1)\end{array}$ & 0 & $\begin{array}{l}1(6.75 \mathrm{E}-05- \\
1.47 \mathrm{E}-02,1)\end{array}$ & 0 & $\begin{array}{l}1(5.58 \mathrm{E}-05- \\
1.22 \mathrm{E}-02,1)\end{array}$ & $\begin{array}{c}3(0.0003- \\
0.0038 \\
0.0712)\end{array}$ \\
\hline
\end{tabular}




\begin{tabular}{|c|c|c|c|c|c|c|c|c|c|}
\hline $\begin{array}{l}\text { NPC1 \& } \\
\text { NPC2 }\end{array}$ & Niemann-Pick disease type C & 0.0063 & $\begin{array}{l}1(5.81 \mathrm{E}-05- \\
1.27 \mathrm{E}-02,1)\end{array}$ & 0 & $\begin{array}{l}1(0.0001- \\
0.0307,1)\end{array}$ & 0 & $\begin{array}{l}1(8.38 \mathrm{E}-05- \\
1.83 \mathrm{E}-02,1)\end{array}$ & 0 & $\begin{array}{l}3(0.0003- \\
0.0038 \\
0.0022)\end{array}$ \\
\hline$P A H$ & Classic phenylketonuria & $2 / 100$ & $\begin{array}{c}4(0.0025- \\
0.0233,0.854)\end{array}$ & $\begin{array}{c}2(0.0004- \\
0.0129 \\
0.0124)\end{array}$ & $\begin{array}{l}1(0.0001- \\
0.0307,1)\end{array}$ & $\begin{array}{c}13(0.0185- \\
0.0585,0.423)\end{array}$ & $\begin{array}{l}2(0.0008- \\
0.0237,1)\end{array}$ & $\begin{array}{l}2(0.0008- \\
0.0237,1)\end{array}$ & $\begin{array}{l}24(0.0067- \\
0.0155 \\
0.0031)\end{array}$ \\
\hline PCCA & Propionic acidemia & 0.00616 & $\begin{array}{l}1(5.81 \mathrm{E}-05- \\
1.27 \mathrm{E}-02,1)\end{array}$ & 0 & 0 & 0 & 0 & 0 & $\begin{array}{l}1(0.0000- \\
0.0024 \\
4.56 \mathrm{E}-05)\end{array}$ \\
\hline PDSS1 & Primary coenzyme Q10 deficiency & unknown & 1 & 0 & 0 & 0 & 0 & 0 & 1 \\
\hline PHGDH & $\begin{array}{c}\text { 3-phosphoglycerate dehydrogenase deficiency, } \\
\text { infantile/juvenile form and/or Neu-Laxova } \\
\text { syndrome }\end{array}$ & $\begin{array}{l}\sim 15 \text { cases } \\
\text { worldwide }\end{array}$ & 0 & 1 & 0 & 1 & 0 & 0 & 2 \\
\hline PSPH & 3-phosphoserine phosphatase deficiency & unknown & 0 & 0 & 0 & 1 & 0 & 0 & 1 \\
\hline PTS & 6-pyruvoyl-tetrahydropterin synthase deficiency & unknown & 1 & 5 & 2 & 7 & 0 & 1 & 16 \\
\hline SGSH & Sanfilippo syndrome type A & 0.0063 & 0 & 0 & $\begin{array}{l}1(0.0001- \\
0.0307,1)\end{array}$ & $\begin{array}{c}4(0.0029- \\
0.0271,0.92)\end{array}$ & 0 & 0 & $\begin{array}{c}5(0.0007- \\
0.0051 \\
0.0235)\end{array}$ \\
\hline SLC25A13 & Citrullinemia type II & 0.0153 & $\begin{array}{l}2(0.0006- \\
0.0165 \\
0.3022)\end{array}$ & 0 & $\begin{array}{l}1(0.0001- \\
0.0307,1)\end{array}$ & $\begin{array}{c}2(0.0006- \\
0.0191,0.555)\end{array}$ & 0 & 0 & $\begin{array}{l}5(0.0007- \\
0.0051 \\
1.81 \mathrm{E}-09)\end{array}$ \\
\hline SLC25A15 & $\begin{array}{l}\text { Hyperornithinemia-hyperammonemia- } \\
\text { homocitrullinuria syndrome }\end{array}$ & $\begin{array}{l}\sim 122 \text { cases } \\
\text { worldwide }\end{array}$ & 1 & 0 & 0 & 3 & 0 & 0 & 4 \\
\hline SLC6A19 & Hartnup disease & 0.0115 & $\begin{array}{l}3(0.0014- \\
0.0200,1)\end{array}$ & $\begin{array}{l}1(4.55 \mathrm{E}-05- \\
9.98 \mathrm{E}-03 \\
0.1029)\end{array}$ & 0 & 0 & $\begin{array}{l}4(0.0036- \\
0.0335,1)\end{array}$ & $\begin{array}{c}2(0.0005- \\
0.0158,0.941)\end{array}$ & $\begin{array}{l}10(0.0021- \\
0.0080 \\
0.0016)\end{array}$ \\
\hline$T H$ & Tyrosine hydroxylase deficiency & $\begin{array}{l}<50 \text { cases } \\
\text { worldwide }\end{array}$ & 0 & 0 & 0 & 0 & 0 & 1 & 1 \\
\hline
\end{tabular}

*Frequency rates for females with X-linked recessive IEM gene variants were calculated based on the disease prevalence, assuming it follows the Hardy Weinberg Equilibrium. For recessive genes, where previously established 
carrier frequency for a given IEM/phenotype was not available, carrier frequency was determined using disease prevalence and the Hardy Weinberg Equilibrium. .* One patient with 2 variants within the recessive gene ASL was calculated as a putative affected patient. All p-values indicated have been Bonferroni corrected and bolded $p$-value indicates a significant overrepresentation of gene variants within the psychiatric patient population relative to the general population. See Supplementary Table S4.2 for the prevalence/carrier rates sources used for statistical comparison. SSD, schizophrenia spectrum disorders; BPD, bipolar disorder; MDD, major depressive disorder; OCD, obsessive compulsive disorder; GAD, generalized anxiety disorder; $\mathrm{P}$, pathogenic; $\mathrm{LP}$, likely pathogenic; $\mathrm{Cl}$, confidence interval; XLD, X-linked dominant gene. 


\subsection{Discussion}

This is the first study to directly investigate the prevalence of a comprehensive list of TGDs that are associated with psychiatric phenotypes in a large primary psychiatric cohort. Overall, 365 unique patients out of 2301 (15.86\%) were identified to carry pathogenic or LP variants in TGD genes; 15 of these patients were found to have multiple variants of interests in genes associated with both dominant and recessive disorders. Of these, 62 (2.69\%) individuals are putatively affected with a TGD, increasing to $72(3.13 \%)$ with the inclusion of female heterozygous OTC and $A B C D 1$ pathogenic variant carriers, in whom variable penetrance and expressivity can affect the rate and severity of clinical presentation (Caldovic et al., 2015; Chongsrisawat et al., 2018; Engelen et al., 2014; Finsterer et al., 2013; Gyato et al., 2004; Schirinzi et al., 2019). A further 23 patients had VUSes that were predicted to cause structural effects on protein products, including 8 in autosomal dominant disorder genes, 2 in X-linked dominant disorder genes, and 13 patients with two variants within the same recessive disorder gene. Including these patients as potentially affected with TGDs would increase the proportion of putatively affected individuals within the psychiatric cohort to $4.13 \%$. Taken together, the prevalence of TGDs in our study cohort is approximately three - and up to four - times the estimated 1\% cumulative prevalence of all single gene disorders globally (Blencowe et al., 2018; WHO, 2020), despite only examining for 108 TGDs. This supports that the prevalence of all genetic disorders, including all single gene and chromosomal disorders, is likely much higher in psychiatric patient populations than previously thought. Additionally, 303 (13.17\%) study patients are putative carriers for one or more of the screened autosomal or X-linked recessive disorders. This includes the discovery of pathogenic/LP variants in genes associated with very rare TGDs (e.g. primary coenzyme Q10 deficiency, cerebral creatine deficiency syndrome 1, among others), suggesting the potential enrichment of these extremely rare genetic diseases within psychiatric patient populations.

There are hundreds of IEMs with a collective prevalence estimated to be 50.9 per 100000 live births (0.051\%) and often overlooked is that many IEMs are treatable 
(Saudubray et al., 2018; Waters et al., 2018). In our previous pilot study (Sriretnakumar et al., 2019), we showed an enrichment of pathogenic variants in genes associated with four treatable IEMs (NPC, homocystinuria due to cystathionine beta-synthase deficiency, Wilson disease and AIP) in a cohort of SSD, BPD and MDD patients, with a putative affected rate of $0.34 \%$ with these four IEMs. . The current study identified 32 patients (1.39\%) putatively affected with an IEM, representing a 27-fold enrichment of a select list of treatable IEMs within our psychiatric cohort relative to the collective prevalence of all IEMs. Major groups of enriched IEMs identified in this study include urea cycle disorders ( $n=49$ affected patients), peroxisomal disorders $(n=10)$ and porphyrias $(n=2)$. Many of these disorders are highly treatable or manageable through diet, medications and lifestyle modifications (Diaz et al., 2013; Fontanellas et al., 2016; Häberle et al., 2019; Jiang et al., 2018; Mew et al., 2017; Morita, 2019; Pischik et al., 2015; S Grewal et al., 2016).

The enrichment of AIP - an autosomal dominant IEM - within the psychiatric population was replicated in this study from our previous study. This finding is in line with numerous studies in the literature demonstrating a strong association between AIP and psychiatric phenotypes (Duque-Serrano et al., 2018). Specifically, one study found a 20-fold increased incidence of AIP in hospitalized psychiatric patients in comparison to the general population, while another study showed AIP patients to be at a four-fold increased risk for being diagnosed with SSD and BPD, and, significantly, their firstdegree relatives were found to have a two-fold increased risk for developing SSD and BPD (Cederlöf et al., 2015; Duque-Serrano et al., 2018; Tishler et al., 1985). Treatment options for the porphyrias include preventing or minimizing acute porphyric crises through avoidance of precipitating factors (e.g. alcohol, smoking, stress, certain medications), which can prevent the onset of psychiatric symptomatology altogether, as well as carbohydrate loading, electrolyte infusions and heme therapy to abrogate acute attacks (Pischik et al., 2015). There are also emerging novel treatments for AIP, including enzyme, gene and messenger RNA-based therapies (Fontanellas et al., 2016; Jiang et al., 2018; Parra-Guillen et al., 2020). 
Urea cycle and peroxisomal disorders, such as the X-linked disorders OTC deficiency and X-ALD, have also been shown to present with psychiatric symptoms akin to SSD, BPD, attention deficit hyperactivity disorder (ADHD) and depression (Kitchin et al., 1987; Shamim et al., 2017; Stepien et al., 2019). OTC deficiency can present in infancy or childhood with seizures, neurodevelopmental impairment and ADHD (LichterKonecki et al., 2016). Although OTC deficiency is classically considered an X-linked recessive disorder, wherein males are affected with more severe phenotypes, it is wellknown that females heterozygous for pathogenic OTC variants are also symptomatic, especially for neuropsychiatric phenotypes with a later onset (Lichter-Konecki et al., 2016; Lipskind et al., 2011; Niwinski et al., 2020; Pridmore et al., 1995). Interestingly, there was no enrichment of female carriers for OTC deficiency within the psychiatric cohort, though this may be confounded by the lack of an established carrier rate with which to accurately compare. Both males and females in the study cohort were enriched for pathogenic variants in $A B C D 1$, the gene associated with X-ALD, in line with the reported $39 \%$ of X-ALD patients who present with one or more psychiatric symptoms, including, most notably, $17 \%$ of patients reported to present with exclusively psychiatric manifestations (Kitchin et al., 1987).

Taken together, the high rate of IEMs found in the study cohort suggests a very strong association between IEMs and psychiatric disorders. Whether the IEMs are causally related or modify existing psychiatric illnesses already present within a given patient is yet to be fully explored. Nevertheless, accurate genetic diagnosis of this subset of psychiatric patients with underlying IEMs is critical to allow for targeted therapy for the underlying disorder. The efficacy of targeted IEM therapies on psychiatric phenotypes specifically requires systematic study. There is clear precedent for positive effects on neuropsychiatric phenotypes from treatment for some common IEMs, such as phenylketonuria and OTC deficiency (Ashe et al., 2019; Gyato et al., 2004; Niwinski et al., 2020).

In this study, carriers for Wilson disease, metachromatic leukodystrophy, NPC and very long chain acyl-CoA dehydrogenase deficiency (VLCADD) were identified, amongst 
many other screened disorders. Heterozygous carriers of autosomal recessive disorders have been shown in previous studies to manifest with clinical findings, including psychiatric phenotypes. There are reports of heterozygous $A T P 7 B$ mutation carriers presenting with neuropsychiatric features that have been alleviated through copper chelation, a standard treatment for full-fledged Wilson disease (Cocco et al., 2009; Tarnacka et al., 2009). Carriers for metachromatic leukodystrophy have been shown to have low arylsulfatase A (ARSA) activity, which has been strongly associated with neuropsychiatric features, including SSD and psychosis; specifically, heterozygous carriers for the ARSA I179S variant present with a psychiatric onset of metachromatic leukodystrophy which can mirror SSD, in addition to presenting with dementia and neurological phenotypes (e.g. paraparesis) (Demily et al., 2014; Ługowska et al., 2005; Marcão et al., 2003). Multiple studies have shown an enrichment for NPC carriers within psychiatric populations (Bauer et al., 2013; Maubert et al., 2013, 2015; Sriretnakumar et al., 2019). One patient with a heterozygous mutation in ACADVL was found to exhibit rhabdomyolysis, a clinical presentation of VLCADD due to biallelic ACADVL mutations (Hisahara et al., 2015). Patients both affected with and carriers for aspartylglucosaminuria have been found to have similar dysmorphic facial features, though no carriers for either disorder have been reported to have psychiatric phenotypes (Arvio et al., 2004). Although it cannot be excluded that a second mutation has been missed based on molecular testing limitations in these patients, it is worthwhile considering the possibility of manifesting carriers, which may pave the way for targeted therapies, as seen in the Wilson disease carrier examples. Future large studies to phenotype and potentially further genotype (e.g. with in-depth search for non-exonic variants) carriers for genetic disorders in psychiatric populations should be considered.

Interestingly, the highest proportion (39\%) of pathogenic and LP variants in the genes studied was found in the OCD subgroup relative to the other psychiatric subgroups in the study cohort, with 20 of 375 OCD patients (5.3\%) putatively affected and $78(20.8 \%)$ carriers for recessive disorders. One potential explanation for this is that 
OCD has one of the most rigorous diagnostic criteria and, thus, the OCD patients within our study sample could have been enriched for more severe presentations of the illness relative to the other psychiatric sub-groups (Association, 2013). This is in line with our previous studies, in which we have shown that psychiatric patients enriched for genetic disease variants present with more severe psychiatric symptomatology in comparison to patients without these variants (Sriretnakumar et al., 2019).

Amongst the genes associated with treatable autosomal recessive disorders screened in this study cohort, there was an enrichment of heterozygous pathogenic/LP variants in COQ9 and GCDH within the OCD subset of patients relative to the general population. Biallelic mutations in COQ9 result in highly heterogeneous primary CoQ10 deficiency, features of which include cognitive deficits, intellectual disability, neuropathy, ataxia and seizures (Adam et al., 1993-2020). Interestingly, deficiency of CoQ10 is hypothesized to contribute to the well-recognized mitochondrial dysfunction seen in neuropsychiatric illnesses, such as SSD, BPD, MDD, Huntington disease and Parkinson's disease (Maguire et al., 2018). CoQ10, an antioxidant, is found to be depleted in patients with neuropsychiatric disorders, and subsequent CoQ10 supplementation has been shown to have antidepressant effects and may slow progression of symptoms in Parkinson's disease (Maguire et al., 2018; Morris et al., 2013). To date, no associations between COQ9 and OCD have been found; however, OCD has been extensively associated with mitochondrial dysfunction and oxidative stress, the same pathways implicated in CoQ10 deficiency (Maia et al., 2019). Specifically, most OCD patients are found to have a dysregulated oxidative profile, wherein the elevated oxidative stress is insufficiently buffered by the antioxidant systems (Maia et al., 2019). Our study suggests a potential association between COQ9 and OCD, which could potentially be mediated through cellular oxidative stress from mitochondrial dysfunction. Further study is warranted, particularly given the possibility of easy and accessible treatment with antioxidants, whether by dietary management or supplementation. 
Biallelic mutations in GCDH result in glutaryl-CoA dehydrogenase deficiency, which leads to a build-up of the neurotoxin glutaric acid, causing GA1 (Goodman et al., 1998; Larson et al., 2019). GA1 can manifest in infancy or have a late onset, and can present with various neurological (e.g. neurodevelopmental impairment, epilepsy, dementia, tremor) and psychiatric (e.g. BPD, anxiety) symptoms (da Costa Ferreira et al., 2008; Goodman et al., 1998; Pokora et al., 2019; Ramsay et al., 2018; Sanju et al., 2020). Glutaric acid has also been identified as a biomarker for violent presentations in SSD (Chen et al., 2020). Although, to date, no direct associations between OCD and GCDH/GA1 have been established, it is of interest to note that late-onset GA1 can present with brain neoplasms and GA1 patients of any age may present with chronic kidney disease (CKD) (Afsoun Seddighi et al., 2015; Larson et al., 2019). A number of studies suggest that brain neoplasms and CKD are associated with higher risk for OCD or OCD-like symptoms (Afsoun Seddighi et al., 2015; Berthier et al., 1996; Chacko et al., 2000; Larson et al., 2019; Yousefichaijan et al., 2014; Yousefichaijan et al., 2016). Several studies have suggested that brain neoplasms and CKD can lead to central nervous system dysfunction, and higher prevalence of psychiatric and cognitive disorders (e.g. memory disorders, anxiety disorders, ADHD, OCD and MDD) (Arnold et al., 2016; Chaijan et al., 2015; Durand et al., 2018; Nur et al., 2019; Silva et al., 2019; Yousefichaijan et al., 2014). However, the exact causal relationships between brain neoplasms, CKD and OCD (and other neuropsychiatric phenotypes) remain unclear (Yousefichaijan et al., 2014; Yousefichaijan et al., 2016). The enrichment of GCDH pathogenic variants in OCD patients should be further explored to elucidate whether there is a true association and its pathobiological mechanism.

An additional OCD patient was found to have 2 LP variants within the $A S L$ gene coding for the enzyme argininosuccinate lyase (ASL), biallelic mutations in which result in autosomal recessive argininosuccinic aciduria (Baruteau et al., 2017). ASL is one of the six enzymes involved in the breakdown and removal of nitrogen; consequently, the main presentation of argininosuccinic aciduria is hyperammonemia (Nagamani et al., 2012). Argininosuccinic aciduria can result in neurodevelopmental impairment, epilepsy, 
seizures and ADHD (Nagamani et al., 2012). To date, there has been one case study of a patient with refractory OCD and body dysmorphic disorder associated with hyperammonemia (Cleveland et al., 2009). Hyperammonemia has been strongly associated with various neuropsychiatric phenotypes, including dementia, psychosis, mood disorders and hallucinations (O. Bonnot et al., 2015; Enns et al., 2005; Leo et al., 2019). Significantly, antipsychotics and mood stabilizers used most commonly in the treatment of SSD and BPD are known to cause recurrent hyperammonemia in patients, resulting in encephalopathy and delirium (Muraleedharan et al., 2015; Y.-F. Wu, 2017). One study showed that up to one-third of SSD patients taking valproic acid were found to have hyperammonemia (Ando et al., 2017). Not surprisingly, administration of valproic acid and corticosteroids can worsen urea cycle disorders, such as argininosuccinic aciduria (O. Bonnot et al., 2015). This underlines the importance of accurate genetic diagnosis in psychiatric patients who may have an underlying genetic disorder for which standard psychotropic medications may be contraindicated. In particular, psychiatric patients presenting with hyperammonemia and/or worsening symptoms following the administration of certain antipsychotics or mood stabilizers should be prioritized for genetic evaluation for a possible IEM.

Genetic variants associated with non-IEM TGDs were also found to be enriched within the study cohort relative to the general population. The majority of these variants were found within ion channel genes associated with autosomal dominant disorders, with a total of 37 patients being putatively affected. Ion channel diseases, known as channelopathies, have been increasingly studied in the field of neuropsychiatry, given that the brain is an electrically excitable tissue (Gargus, 2006; Schmunk et al., 2013). Channelopathies result from mutations in calcium, sodium, potassium and/or chloride ion channels, all of which are involved in a wide array of physiological processes, including neurotransmission, secretion and cell proliferation, among others (Imbrici et al., 2016). Channelopathies affecting the central nervous system include cerebellar ataxia syndromes, epileptic encephalopathies and familial hemiplegic migraine (Imbrici et al., 2016). Interestingly, channelopathy phenotypes such as epilepsy are also comorbid for 
other neurodevelopmental disorders, including autism spectrum disorder (ASD), intellectual disability, parkinsonism, SSD, BPD and others (Fanella et al., 2020; Imbrici et al., 2016; Knott et al., 2016). Most importantly, channelopathies can respond to specific medications that modulate ion channel activity, such as acetazolamide or valproate (Camia et al., 2017; Chiron et al., 2011; Cleland et al., 2008; Imbrici et al., 2016). Novel therapies, including gene therapy, gene editing and animal toxins, are also being explored for the treatment of channelopathies (Kozlov, 2018; Wykes et al., 2018).

One of the genes with the most variants found within the study sample $(n=20)$ was CACNA1A, which encodes the calcium voltage-gated channel subunit alpha-1 (Indelicato et al., 2019). Mutations in CACNA1A result in dominantly inherited familial hemiplegic migraine, epileptic encephalopathy, episodic ataxia type 2 and/or spinocerebellar ataxia type 6 phenotypes (Indelicato et al., 2019). Furthermore, CACNA1A variants have been associated with ASD, SSD and BPD in numerous psychiatric genetic studies (Damaj et al., 2015; Indelicato et al., 2019; Li et al., 2017; McCarthy et al., 2016). Interestingly, variants in CACNA1A have been shown to play a role in antipsychotic treatment response in SSD patients (O'Connell et al., 2019). There were also numerous variants $(\mathrm{n}=18)$ identified within the voltage-gated sodium channel genes, SCN1A, SCN2A and SCN3A. Mutations in the SCN genes are associated with autosomal dominant generalized epilepsy with febrile seizures, Dravet syndrome and/or familial hemiplegic migraine (Miller et al., 2019; Wolff et al., 2017; Zaman et al., 2020). There is also increasing evidence for the association of SCN1A, SCN2A and SCN3A variants with ASD, SSD and BPD, with genotype-phenotype correlations showing that the functional effect of a given mutation tends to be associated with a specific set of clinical presentations (Bartnik et al., 2011; Carroll et al., 2016; Nickel et al., 2018; Suddaby et al., 2019; Yamakawa, 2016).. For example, SCN2A loss-of-function mutations are generally associated with neurological phenotypes (e.g. ataxia, epilepsy), while gainof-function mutations tend to be associated with neuropsychiatric phenotypes (e.g. ASD, intellectual disability), and some variants with both gain- and loss-of-function effects can result in a broader phenotypic spectrum (Suddaby et al., 2019; Winquist et 
al., 2018). Furthermore, using gene set-based analytics testing on genome-wide association study data, Askland et al. (2012) found ion channel genes to be consistently enriched in SSD samples across both European-American and African-American ethnic groups, suggesting that variations within ion channel genes could play a role in SSD genetic susceptibility (Askland et al., 2012). In this study, 37 patients were identified to be putatively affected with a channelopathy (one patient had two different CACNA1A variants), with one-third ( $\mathrm{n}=12$ ) diagnosed with SSD, mirroring the known strong association of ion channel dysfunction and SSD susceptibility. Of the SSD patients, four patients were identified to have CACN1A1 pathogenic/LP variants. Although there was no direct measure of treatment-responsiveness in these patients, 3 (25\%) had reportedly been treated with clozapine or olanzapine, which are often second-line antipsychotics used after incomplete response to first-line antipsychotics. This provides further support for the role of voltage-gated calcium channel gene variants in SSD treatment response (O'Connell et al., 2019).

Of interest, another third $(n=11)$ of the putative channelopathy patients were diagnosed with OCD. Although there is no direct association known between $C A C N A 1 A$ and SCN gene variants, and OCD, there is some literature suggesting a potential link between ion channel genes and OCD-like symptoms/behaviours. Specifically, familial hemiplegic migraine patients and mouse models with $C A C N A 1 A$ mutations have been reported to present with comorbid OCD or OCD-like behaviours (Bøttger et al., 2012; Bøttger et al., 2016; Dehghani et al., 2019; Marconi et al., 2003; Santoro et al., 2011). Interestingly, calcium channel antagonists showed inhibition of OCD behaviours in OCD mouse models and had anxiolytic effects (Bandelow, 2008; Egashira et al., 2008), while sodium channel activators have been shown to induce anxiety- and OCD-like behaviours in rats (Saitoh et al., 2015). These studies support a potential shared molecular pathophysiology between ion channel disorders and OCD-related phenotypes.

Two patients were found to be putatively affected with a channelopathy, as well as another genetic disease. Specifically, one male OCD patient was found to have 
pathogenic variants in both SCN1A and OTC, while a female SSD patient was found to have pathogenic variants in SCN1A and PDHA1. Interestingly, OTC deficiency patients can present with cerebellar ataxia, seizures and epilepsy, all of which are phenotypes also prevalent amongst channelopathies (Barkovich et al., 2020; Crowe et al., 2018; Hidaka et al., 2020; Im et al., 2018; Pizzi et al., 2019; B. Wu et al., 2018). Moreover, OTC deficiency is considered a differential diagnosis for hemiplegic migraine (Kumar et al., 2020). Similarly, PDHA1 deficiency patients can also present with neurological phenotypes akin to channelopathies (Bhandary et al., 2015; Debray et al., 2008; Prasad et al., 2011). Even more striking is the finding of anti-PDHA1 antibodies in a subset of SSD patients (Nakagami et al., 2020). Nakagami et al. (2020) found that patients with antiPDHA1 antibodies had increased volumes of the left occipital fusiform gyrus and left cuneus, a finding that is in contrast to the decreased volumes typically seen in SSD patients in comparison to controls (Nakagami et al., 2020). Increased volumes of fusiform gyrus have been previously associated with synesthesia - a disorder characterized by abnormal perception in response to the presence or absence of an external sensory stimulus - which can potentially be linked to an underlying common mechanism for hallucinations and delusions typical of SSD (Bouvet et al., 2017; Hupé et al., 2015; Weiss et al., 2009). PDHA1 dysfunction has been associated with mitochondrial dysfunction, as well as numerous neuropsychiatric disorders, including Parkinson's disease, dementia, psychosis and SSD (Nakagami et al., 2020). A patient with PDHA1 mutation was diagnosed with SSD after presenting with auditory hallucinations, psychosis and seizures, suggesting the potential for a subgroup of SSD patients to be influenced by genetic disorders that can cause organic psychosis (Satogami et al., 2017). Most notably, PDHA1 deficiency patients have been shown to have favorable response to acetazolamide, a drug commonly used to treat channelopathies (Egel et al., 2010; Livingstone et al., 1984; Platt et al., 2012).

Taken together, there is a growing body of evidence for shared pathogenetic mechanisms of channelopathies and psychiatric disorders, with important implications for development of targeted therapies that can potentially be effective across the 
various brain phenotypes associated with ion channel genes (Gargus, 2006; Imbrici et al., 2016). This further emphasizes the need for accurate genetic diagnosis of psychiatric patients so that precision therapies can be initiated.

A major limitation of our study was the paucity of clinical phenotypic data beyond the psychiatric diagnoses and medications taken at the time of recruitment. Statistical analysis of this data did not reveal any differences in the average number of medications for patients with and without genetic disease variants (data not shown). We were not able to postulate based on the available clinical data whether patients had non-psychiatric findings consistent with the genetic disorders identified in this study. However, all patients included in this study consented to be re-contacted, enabling future follow-up to clinically validate the study findings. Another limitation of the study analysis was the assumption of HWE in calculating carrier frequencies. Comparing the established rate of female heterozygous $A B C D 1$ mutation carriers at $9.52 \mathrm{E}-06$ to the calculated rate of 4.5E-03 based on HWE, it can be seen that the calculated rate is significantly higher than the observed heterozygote rate (Wiesinger et al., 2015). Similarly, the calculation of rates of females heterozygous for OTC mutations and carrier rates for recessive disorders where established carrier rates were not known/available based on HWE could have led to an inflated estimation. This may contribute to the apparent lack of enrichment of variants in these genes in analysis of the study results. Finally, future replication studies should include carefully clinically screened control samples for direct statistical comparison purposes.

In conclusion, this is the first direct, comprehensive study of the burden of TGDs in a large, varied psychiatric cohort. The results of this study support that pathogenic gene variants associated with TGDs are enriched in primary psychiatric populations. This strongly suggests that the prevalence of these, and most likely many other, genetic diseases are greatly underestimated in psychiatric populations. Increasing awareness and ensuring accurate diagnosis of TGDs will open new avenues to targeted treatment for a subset of psychiatric patients. Future studies into the psychiatric sub-phenotypes associated with TGDs and whether carriers of these disorders may exhibit clinical 
manifestations, as well as the response of psychiatric symptomatology to targeted treatments will be vital in optimizing the precision diagnosis and management of many psychiatric patients.

\subsection{Acknowledgements}

The authors would like to thank Dr. John B. Vincent, Dr. Clement Zai, and Anna Mikhailov for their technical assistance, and critical and constructive input during this study.

\subsection{Conflict of Interest}

All authors declare no conflict of interest.

\subsection{Funding Information}

V.S. was funded by the Canadian Institute of Health Research (CIHR) doctoral award. This project was funded by an unrestricted research grant from Recordati Rare Diseases.

\subsection{References}

Adam, M., Ardinger, H., Pagon, R., Wallace, S., Bean, L., Stephens, K., \& Amemiya, A. (19932020). Primary Coenzyme Q10 Deficiency--GeneReviews®.

Afsoun Seddighi, M., Seddighi, A. S., Amir Nikouei, M., Farzad Ashrafi, M., \& Nohesara, S. (2015). Psychological aspects in brain tumor patients: A prospective study. Hell J Nucl Med, 64.

Ando, M., Amayasu, H., Itai, T., \& Yoshida, H. (2017). Association between the blood concentrations of ammonia and carnitine/amino acid of schizophrenic patients treated with valproic acid. BioPsychoSocial medicine, 11(1), 19.

Arnold, R., Issar, T., Krishnan, A. V., \& Pussell, B. A. (2016). Neurological complications in chronic kidney disease. JRSM cardiovascular disease, 5, 2048004016677687.

Arvio, M. A., Peippo, M. M., Arvio, P. J., \& Kääriäinen, H. A. (2004). Dysmorphic facial features in aspartylglucosaminuria patients and carriers. Clinical dysmorphology, 13(1), 11-15. 
Ashe, K., Kelso, W., Farrand, S., Panetta, J., Fazio, T., De Jong, G., \& Walterfang, M. (2019). Psychiatric and Cognitive Aspects of Phenylketonuria: The Double-Edged Sword of Diet and Promise of New Treatments. Front Psychiatry, 10, 561.

Askland, K., Read, C., O’Connell, C., \& Moore, J. H. (2012). Ion channels and schizophrenia: a gene set-based analytic approach to GWAS data for biological hypothesis testing. Human Genetics, 131(3), 373-391.

Association, A. P. (2013). Diagnostic and statistical manual of mental disorders (DSM-5®): American Psychiatric Pub.

Bandelow, B. (2008). The medical treatment of obsessive-compulsive disorder and anxiety. CNS spectrums, 13(S14), 37-46.

Baraban, S. C., Dinday, M. T., \& Hortopan, G. A. (2013). Drug screening in Scn1a zebrafish mutant identifies clemizole as a potential Dravet syndrome treatment. Nature communications, 4(1), 1-10.

Barkovich, E., \& Gropman, A. (2020). Late Onset Ornithine Transcarbamylase Deficiency Triggered by an Acute Increase in Protein Intake: A Review of 10 Cases Reported in the Literature. Case Reports in Genetics, 2020.

Bartnik, M., Chun-Hui Tsai, A., Xia, Z., Cheung, S., \& Stankiewicz, P. (2011). Disruption of the SCN2A and SCN3A genes in a patient with mental retardation, neurobehavioral and psychiatric abnormalities, and a history of infantile seizures. Clinical genetics, $80(2)$, 191-195.

Baruteau, J., Jameson, E., Morris, A. A., Chakrapani, A., Santra, S., Vijay, S., . . Murphy, E. (2017). Expanding the phenotype in argininosuccinic aciduria: need for new therapies. Journal of Inherited Metabolic Disease: Official Journal of the Society for the Study of Inborn Errors of Metabolism, 40(3), 357-368.

Bauer, P., Balding, D. J., Klünemann, H. H., Linden, D. E., Ory, D. S., Pineda, M., . . ChadhaBoreham, H. (2013). Genetic screening for Niemann-Pick disease type $\mathrm{C}$ in adults with neurological and psychiatric symptoms: findings from the ZOOM study. Hum Mol Genet, 4349-4356.

Berthier, M. L., Kulisevsky, J., Gironell, A., \& Heras, J. A. (1996). Obsessive-compulsive disorder associated with brain lesions: clinical phenomenology, cognitive function, and anatomic correlates. Neurology, 47(2), 353-361.

Bhandary, S., \& Aguan, K. (2015). Pyruvate dehydrogenase complex deficiency and its relationship with epilepsy frequency-An overview. Epilepsy Res, 116, 40-52.

Blencowe, H., Moorthie, S., Petrou, M., Hamamy, H., Povey, S., Bittles, A., . . Modell, B. (2018). Rare single gene disorders: estimating baseline prevalence and outcomes worldwide. Journal of community genetics, 9(4), 397-406. 
Bokma, W. A., Wetzer, G. A., Gehrels, J. B., Penninx, B. W., Batelaan, N. M., \& van Balkom, A. L. (2019). Aligning the many definitions of treatment resistance in anxiety disorders: A systematic review. Depression and anxiety, 36(9), 801-812.

Bonnot, O., Gama, C. S., Mengel, E., Pineda, M., Vanier, M. T., Watson, L., . . Patterson, M. C. (2019). Psychiatric and neurological symptoms in patients with Niemann-pick disease type C (NP-C): findings from the international NPC registry. The World Journal of Biological Psychiatry, 20(4), 310-319.

Bonnot, O., Herrera, P. M., Tordjman, S., \& Walterfang, M. (2015). Secondary psychosis induced by metabolic disorders. Front Neurosci, 9, 177. doi: 10.3389/fnins.2015.00177

Bøttger, P., Doğanlı, C., \& Lykke-Hartmann, K. (2012). Migraine-and dystonia-related diseasemutations of $\mathrm{Na}+\mathrm{K}+-\mathrm{ATPases}$ : relevance of behavioral studies in mice to disease symptoms and neurological manifestations in humans. Neuroscience \& Biobehavioral Reviews, 36(2), 855-871.

Bøttger, P., Glerup, S., Gesslein, B., Illarionova, N. B., Isaksen, T. J., Heuck, A., . . Gunnarson, E. (2016). Glutamate-system defects behind psychiatric manifestations in a familial hemiplegic migraine type 2 disease-mutation mouse model. Scientific Reports, 6(1), 1-21.

Bouvet, L., Barbier, J.-E., Cason, N., Bakchine, S., \& Ehrlé, N. (2017). When synesthesia and savant abilities are mistaken for hallucinations and delusions: contribution of a cognitive approach for their differential diagnosis. The Clinical Neuropsychologist, 31(8), 14591473.

Cabal-Herrera, A. M., Saldarriaga-Gil, W., Salcedo-Arellano, M. J., \& Hagerman, R. J. (2020). Fragile X associated neuropsychiatric disorders in a male without FXTAS. Intractable \& Rare Diseases Research.

Caldovic, L., Abdikarim, I., Narain, S., Tuchman, M., \& Morizono, H. (2015). Genotypephenotype correlations in ornithine transcarbamylase deficiency: A mutation update. Journal of Genetics and Genomics, 42(5), 181-194.

Camia, F., Pisciotta, L., Morana, G., Schiaffino, M. C., Renna, S., Carrera, P., . . Siri, L. (2017). Combined early treatment in hemiplegic attacks related to CACNA1A encephalopathy with brain oedema: Blocking the cascade? Cephalalgia, 37(12), 1202-1206.

Carroll, L. S., Woolf, R., Ibrahim, Y., Williams, H. J., Dwyer, S., Walters, J., . . Owen, M. J. (2016). Mutation screening of SCN2A in schizophrenia and identification of a novel lossof-function mutation. Psychiatr Genet, 26(2), 60.

Cederlöf, M., Bergen, S. E., Larsson, H., Landen, M., \& Lichtenstein, P. (2015). Acute intermittent porphyria: comorbidity and shared familial risks with schizophrenia and bipolar disorder in Sweden. The British Journal of Psychiatry, 207(6), 556-557. 
Chacko, R. C., Corbin, M. A., \& Harper, R. G. (2000). Acquired obsessive-compulsive disorder associated with basal ganglia lesions. The Journal of neuropsychiatry and clinical neurosciences, 12(2), 269-272.

Chaijan, P. Y., Sharafkhah, M., Salehi, B., \& Rafiei, M. (2015). Attention deficit hyperactivity disorder in children with early stages of chronic kidney disease. Medical journal of the Islamic Republic of Iran, 29, 181.

Charney, A. W., Stahl, E. A., Green, E. K., Chen, C.-Y., Moran, J. L., Chambert, K., . . Lee, P. H. (2019). Contribution of rare copy number variants to bipolar disorder risk is limited to schizoaffective cases. Biological psychiatry, 86(2), 110-119.

Chen, X., Xu, J., Tang, J., Dai, X., Huang, H., Cao, R., \& Hu, J. (2020). Dysregulation of amino acids and lipids metabolism in schizophrenia with violence. BMC psychiatry, 20(1), 1-11.

Chiron, C., \& Dulac, O. (2011). The pharmacologic treatment of Dravet syndrome. Epilepsia, $52,72-75$.

Chongsrisawat, V., Damrongphol, P., Ittiwut, C., Ittiwut, R., Suphapeetiporn, K., \& Shotelersuk, V. (2018). The phenotypic and mutational spectrum of Thai female patients with ornithine transcarbamylase deficiency. Gene, 679, 377-381.

Cleland, J. C., \& Griggs, R. C. (2008). Treatment of neuromuscular channelopathies: current concepts and future prospects. Neurotherapeutics, 5(4), 607-612.

Cleveland, W. L., DeLaPaz, R. L., Fawwaz, R. A., \& Challop, R. S. (2009). High-dose glycine treatment of refractory obsessive-compulsive disorder and body dysmorphic disorder in a 5-year period. Neural Plast, 2009, 768398. doi: 10.1155/2009/768398

Cleynen, I., Engchuan, W., Hestand, M. S., Heung, T., Holleman, A. M., Johnston, H. R., . . . Morrow, B. E. (2020). Genetic contributors to risk of schizophrenia in the presence of a 22q11. 2 deletion. Molecular psychiatry, 1-15.

Cocco, G. A., Loudianos, G., Pes, G. M., Tolu, F., Lepori, M. B., Barrocu, M., \& Sechi, G. P. (2009). "Acquired" hepatocerebral degeneration in a patient heterozygote carrier for a novel mutation in ATP7B gene. Movement Disorders, 24(11), 1706-1708.

Crowe, L., Anderson, V., Hardikar, W., \& Boneh, A. (2018). Cognitive and behavioural outcomes of paediatric liver transplantation for ornithine transcarbamylase deficiency JIMD Reports, Volume 43 (pp. 19-25): Springer.

da Costa Ferreira, G., Schuck, P. F., Viegas, C. M., Tonin, A., Ribeiro, C. A. J., Pettenuzzo, L. F., . . Wajner, M. (2008). Chronic early postnatal glutaric acid administration causes cognitive deficits in the water maze. Behavioural brain research, 187(2), 411-416.

Damaj, L., Lupien-Meilleur, A., Lortie, A., Riou, É., Ospina, L. H., Gagnon, L., . . Rossignol, E. (2015). CACNA1A haploinsufficiency causes cognitive impairment, autism and 
epileptic encephalopathy with mild cerebellar symptoms. European Journal of Human Genetics, 23(11), 1505-1512.

Debray, F.-G., Lambert, M., Gagne, R., Maranda, B., Laframboise, R., MacKay, N., . . . Mitchell, G. (2008). Pyruvate dehydrogenase deficiency presenting as intermittent isolated acute ataxia. Neuropediatrics, 39(1), 20-23.

Dehghani, A., \& Karatas, H. (2019). Mouse Models of Familial Hemiplegic Migraine for Studying Migraine Pathophysiology. Current neuropharmacology, 17(10), 961-973.

Demily, C., Parant, F., Cheillan, D., Broussolle, E., Pavec, A., Guillaud, O., . . . Bost, M. (2017). Screening of Wilson's disease in a psychiatric population: difficulties and pitfalls. A preliminary study. Annals of General Psychiatry, 16(1), 1-8.

Demily, C., \& Sedel, F. (2014). Psychiatric manifestations of treatable hereditary metabolic disorders in adults. Annals of General Psychiatry, 13(1), 27.

Diaz, G. A., Krivitzky, L. S., Mokhtarani, M., Rhead, W., Bartley, J., Feigenbaum, A., . . . Gallagher, R. (2013). Ammonia control and neurocognitive outcome among urea cycle disorder patients treated with glycerol phenylbutyrate. Hepatology, 57(6), 2171-2179.

Duque-Serrano, L., Patarroyo-Rodriguez, L., Gotlib, D., \& Molano-Eslava, J. C. (2018). Psychiatric aspects of acute porphyria: a comprehensive review. Current psychiatry reports, 20(1), 5 .

Durand, T., Berzero, G., Bompaire, F., Hoffmann, S., Léger, I., Jego, V., . . Psimaras, D. (2018). Episodic memory impairments in primary brain tumor patients. Archives of Clinical Neuropsychology, 33(8), 949-955.

Egashira, N., Okuno, R., Abe, M., Matsushita, M., Mishima, K., Iwasaki, K., . . Fujiwara, M. (2008). Calcium-channel antagonists inhibit marble-burying behavior in mice. $J$ Pharmacol Sci, 108(1), 140-143.

Egel, R. T., Hoganson, G. E., Katerji, M. A., \& Borenstein, M. J. (2010). Zonisamide ameliorates symptoms of secondary paroxysmal dystonia. Pediatric neurology, 43(3), 205-208.

Engelen, M., Barbier, M., Dijkstra, I. M., Schür, R., de Bie, R. M., Verhamme, C., . . van Geel, B. M. (2014). X-linked adrenoleukodystrophy in women: a cross-sectional cohort study. Brain, 137(3), 693-706.

Enns, G. M., O'Brien, W. E., Kobayashi, K., Shinzawa, H., \& Pellegrino, J. E. (2005). Postpartum "psychosis" in mild argininosuccinate synthetase deficiency. Obstetrics \& Gynecology, 105(5), 1244-1246.

Fabbri, C., Kasper, S., Kautzky, A., Bartova, L., Dold, M., Zohar, J., . . Raimondi, I. (2019). Genome-wide association study of treatment-resistance in depression and meta-analysis of three independent samples. The British Journal of Psychiatry, 214(1), 36-41. 
Fanella, M., Frascarelli, M., Lambiase, C., Morano, A., Unolt, M., Liberati, N., . . Ceccanti, M. (2020). Myoclonic epilepsy, parkinsonism, schizophrenia and left-handedness as common neuropsychiatric features in 22q11. 2 deletion syndrome. J Med Genet, 57(3), 151-159.

Finsterer, J., Lässer, S., \& Stöphasius, E. (2013). Dementia from the ABCD1 mutation c. 1415$1416 \mathrm{del} A \mathrm{G}$ in a female carrier. Gene, 530(1), 155-157.

Fontanellas, A., Avila, M. A., \& Berraondo, P. (2016). Emerging therapies for acute intermittent porphyria. Expert reviews in molecular medicine, 18.

Gargus, J. J. (2006). Ion channel functional candidate genes in multigenic neuropsychiatric disease. Biological psychiatry, 60(2), 177-185.

Goodman, S. I., Stein, D. E., Schlesinger, S., Christensen, E., Schwartz, M., Greenberg, C. R., \& Elpeleg, O. N. (1998). Glutaryl-CoA dehydrogenase mutations in glutaric acidemia (type I): review and report of thirty novel mutations. Human mutation, 12(3), 141-144.

Gordovez, F. J. A., \& McMahon, F. J. (2020). The genetics of bipolar disorder. Molecular psychiatry, 1-16.

Gyato, K., Wray, J., Huang, Z., Yudkoff, M., \& Batshaw, M. L. (2004). Metabolic and neuropsychological phenotype in women heterozygous for ornithine transcarbamylase deficiency. Annals of Neurology: Official Journal of the American Neurological Association and the Child Neurology Society, 55(1), 80-86.

Häberle, J., Burlina, A., Chakrapani, A., Dixon, M., Karall, D., Lindner, M., . . Santer, R. (2019). Suggested guidelines for the diagnosis and management of urea cycle disorders: first revision. Journal of inherited metabolic disease, 42(6), 1192-1230.

Herbert, D., Neves-Pereira, M., Baidya, R., Cheema, S., Groleau, S., Shahmirian, A., . . Müller, D. J. (2018). Genetic testing as a supporting tool in prescribing psychiatric medication: Design and protocol of the IMPACT study. Journal of Psychiatric Research, 96, 265272.

Hidaka, M., Higashi, E., Uwatoko, T., Uwatoko, K., Urashima, M., Takashima, H., . . . Sugimori, H. (2020). Late-onset ornithine transcarbamylase deficiency: a rare cause of recurrent abnormal behavior in adults. Acute Medicine \& Surgery, 7(1), e565.

Hisahara, S., Matsushita, T., Furuyama, H., Tajima, G., Shigematsu, Y., Imai, T., \& Shimohama, S. (2015). A heterozygous missense mutation in adolescent-onset very long-chain acylCoA dehydrogenase deficiency with exercise-induced rhabdomyolysis. The Tohoku journal of experimental medicine, 235(4), 305-310.

Hupé, J.-M., \& Dojat, M. (2015). A critical review of the neuroimaging literature on synesthesia. Frontiers in human neuroscience, 9, 103. 
Im, M., Song, A., Lee, S.-Y., Park, H.-D., Cho, S. Y., \& Jin, D.-k. (2018). A Case of Ornithine Transcarbamylase Deficiency in a Boy with Neonatal Seizure and Altered Mentality. Journal of The Korean Society of Inherited Metabolic disease, 18(2), 55-61.

Imbrici, P., Liantonio, A., Camerino, G. M., De Bellis, M., Camerino, C., Mele, A., . . . Tricarico, D. (2016). Therapeutic approaches to genetic ion channelopathies and perspectives in drug discovery. Frontiers in pharmacology, 7, 121.

IMPACT. (2017). Take part in the study: Participants. from http://impact.camhx.ca/en/participants

Indelicato, E., Nachbauer, W., Karner, E., Eigentler, A., Wagner, M., Unterberger, I., . . . Boesch, S. (2019). The neuropsychiatric phenotype in CACNA1A mutations: a retrospective single center study and review of the literature. European Journal of Neurology, 26(1), 66-e67.

Jiang, L., Berraondo, P., Jericó, D., Guey, L. T., Sampedro, A., Frassetto, A., . . Alegre, M. (2018). Systemic messenger RNA as an etiological treatment for acute intermittent porphyria. Nature medicine, 24(12), 1899-1909.

Jitpimolmard, N., Matthews, E., \& Fialho, D. (2020). Treatment Updates for Neuromuscular Channelopathies. Current Treatment Options in Neurology, 22(10), 1-20.

Kitchin, W., Cohen-Cole, S. A., \& Mickel, S. F. (1987). Adrenoleukodystrophy: frequency of presentation as a psychiatric disorder. Biological psychiatry, 22(11), 1375-1387.

Kleopa, K. (2011). Autoimmune channelopathies of the nervous system. Current neuropharmacology, 9(3), 458-467.

Knott, S., Forty, L., Kerr, M., Gordon-Smith, K., Jones, L., Craddock, N., \& Jones, I. (2016). Epilepsy in bipolar disorder: impact on clinical features, course and outcome. Bipolar disorders, $18(\mathrm{~S} 1)$.

Knupp, K. G., \& Wirrell, E. C. (2018). Treatment strategies for Dravet syndrome. CNS drugs, $32(4), 335-350$.

Kozlov, S. (2018). Animal toxins for channelopathy treatment. Neuropharmacology, 132, 83-97.

Kumar, A., Samanta, D., Emmady, P. D., \& Arora, R. (2020). Hemiplegic migraine. StatPearls [Internet].

Landrum, M. J., Lee, J. M., Benson, M., Brown, G. R., Chao, C., Chitipiralla, S., . . Maglott, D. R. (2018). ClinVar: improving access to variant interpretations and supporting evidence. Nucleic Acids Res, 46(D1), D1062-d1067. doi: 10.1093/nar/gkx1153

Larson, A., \& Goodman, S. (2019). Glutaric Acidemia Type 1 GeneReviews ${ }^{\circledR}[$ Internet]: University of Washington, Seattle. 
Leo, C., Wang, Y., Mold, A., Quintana, J., Shi, H., Abdullah, M., . . Petrillo, R. (2019). Noncirrhotic hyperammonemia: A factor behind dementia to alter mental status. Clinical case reports, 7(11), 2118-2122.

Li, Z., Chen, J., Yu, H., He, L., Xu, Y., Zhang, D., . . Shen, J. (2017). Genome-wide association analysis identifies 30 new susceptibility loci for schizophrenia. Nature genetics, 49(11), 1576.

Lichter-Konecki, U., Caldovic, L., Morizono, H., \& Simpson, K. (2016). Ornithine transcarbamylase deficiency GeneReviews ${ }^{\circledR}$ [Internet]: University of Washington, Seattle.

Lipskind, S., Loanzon, S., Simi, E., \& Ouyang, D. (2011). Hyperammonemic coma in an ornithine transcarbamylase mutation carrier following antepartum corticosteroids. Journal of Perinatology, 31(10), 682-684.

Litwin, T., Dzieżyc, K., \& Członkowska, A. (2019). Wilson disease-treatment perspectives. Annals of translational medicine, 7(Suppl 2).

Liu, C., Kanazawa, T., Tian, Y., Saini, S. M., Mancuso, S., Mostaid, M. S., . . Yu, H. (2019). The schizophrenia genetics knowledgebase: a comprehensive update of findings from candidate gene studies. Translational psychiatry, 9(1), 1-7.

Livingstone, I., Gardner-Medwin, D., \& Pennington, R. (1984). Familial intermittent ataxia with possible X-linked recessive inheritance: two patients with abnormal pyruvate metabolism and a response to acetazolamide. Journal of the neurological sciences, 64(1), 89-97.

Ługowska, A., Berger, J., Tylki-Szymańska, A., Löschl, B., Molzer, B., Zobel, M., \& Czartoryska, B. (2005). Molecular and phenotypic characteristics of metachromatic leukodystrophy patients from Poland. Clinical genetics, 68(1), 48-54.

Maguire, Á., Hargreaves, A., \& Gill, M. (2018). Coenzyme Q10 and neuropsychiatric and neurological disorders: relevance for schizophrenia. Nutritional neuroscience, 1-14.

Maia, A., Oliveira, J., Lajnef, M., Mallet, L., Tamouza, R., Leboyer, M., \& Oliveira-Maia, A. J. (2019). Oxidative and nitrosative stress markers in obsessive-compulsive disorder: a systematic review and meta-analysis. Acta Psychiatrica Scandinavica, 139(5), 420-433.

Marcão, A., Pinto, E., Rocha, S., Miranda, M. S., Ferreira, L. s., \& Amaral, O. (2003). ARSAPD associated alleles in the Portuguese population: frequency determination and haplotype analysis. Mol Genet Metab, 79(4), 305-307.

Marconi, R., De Fusco, M., Aridon, P., Plewnia, K., Rossi, M., Carapelli, S., . . Epifanio, A. (2003). Familial hemiplegic migraine type 2 is linked to $0.9 \mathrm{Mb}$ region on chromosome 1q23. Annals of Neurology: Official Journal of the American Neurological Association and the Child Neurology Society, 53(3), 376-381. 
Maubert, A., Hanon, C., \& Metton, J. (2013). Adult onset Niemann-Pick type C disease and psychosis: literature review. Encephale, 39(5), 315.

Maubert, A., Hanon, C., \& Metton, J. (2015). Niemann-pick type C disease and psychosis: two siblings. Encephale, 41(3), 238-243.

McCarthy, M. J., Le Roux, M. J., Wei, H., Beesley, S., Kelsoe, J. R., \& Welsh, D. K. (2016). Calcium channel genes associated with bipolar disorder modulate lithium's amplification of circadian rhythms. Neuropharmacology, 101, 439-448.

Meier, S. M., \& Deckert, J. (2019). Genetics of anxiety disorders. Curr Psychiatry Rep, 21(3), 16.

Mew, N. A., Simpson, K. L., Gropman, A. L., Lanpher, B. C., Chapman, K. A., \& Summar, M. L. (2017). Urea cycle disorders overview GeneReviews ${ }^{\circledR}$ [Internet]: University of Washington, Seattle.

Middleton, R., Wheaton, M. G., Kayser, R., \& Simpson, H. B. (2019). Treatment resistance in obsessive-compulsive disorder Treatment Resistance in Psychiatry (pp. 165-177): Springer.

Miller, I. O., \& de Menezes, M. A. S. (2019). SCN1A seizure disorders GeneReviews ${ }^{\circledR}$ [Internet]: University of Washington, Seattle.

Mizuno, Y., McCutcheon, R. A., Brugger, S. P., \& Howes, O. D. (2020). Heterogeneity and efficacy of antipsychotic treatment for schizophrenia with or without treatment resistance: a meta-analysis. Neuropsychopharmacology, 45(4), 622-631.

Morita, M. (2019). Therapeutic Strategies for X-Linked Adrenoleukodystrophy, a Representative Peroxisomal Disorder Peroxisomes: Biogenesis, Function, and Role in Human Disease (pp. 171-200): Springer.

Morris, G., Anderson, G., Berk, M., \& Maes, M. (2013). Coenzyme Q10 depletion in medical and neuropsychiatric disorders: potential repercussions and therapeutic implications. Molecular neurobiology, 48(3), 883-903.

Muraleedharan, A., Palappallil, D. S., Gangadhar, R., \& Das, S. (2015). Valproate Induced Hyperammonemic Delirium. J Clin Diagn Res, 9(12), Fr01-03. doi: $10.7860 / \mathrm{jcdr} / 2015 / 15562.6883$

Nagamani, S. C., Erez, A., \& Lee, B. (2012). Argininosuccinate lyase deficiency. Genetics in Medicine, 14(5), 501-507.

Nakagami, Y., Sugihara, G., Nakashima, N., Hazama, M., Son, S., Ma, S., . . Murakami, K. (2020). Anti-PDHA1 antibody is detected in a subset of patients with schizophrenia. Scientific Reports, 10(1), 1-12. 
Nickel, K., van Elst, L. T., Domschke, K., Gläser, B., Stock, F., Endres, D., . . Riedel, A. (2018). Heterozygous deletion of SCN2A and SCN3A in a patient with autism spectrum disorder and Tourette syndrome: a case report. BMC psychiatry, 18(1), 248.

Niwinski, P., Remberk, B., Rybakowski, F., \& Rokicki, D. (2020). Psychiatric Symptoms as the First or Solitary Manifestation of Somatic Illnesses: Hyperammonaemia Type II. Neuropsychobiology, 1-5.

Northrup, H., Koenig, M. K., Pearson, D. A., \& Au, K. S. (2018). Tuberous sclerosis complex GeneReviews ${ }^{\circledR}$ [Internet]: University of Washington, Seattle.

Nur, N., \& Harun, M. (2019). Variation in Neoplasm in Relation with Production of Different Types of Obsession and Compulsion. Cancer Clin J, 1(1), 1005.

O'Connell, K., McGregor, N., \& Warnich, L. (2019). VARIATION IN VOLTAGE-GATED CALCIUM CHANNEL GENES IS ASSOCIATED WITH ANTIPSYCHOTIC TREATMENT RESPONSE IN A SOUTH AFRICAN FIRST EPISODE SCHIZOPHRENIA COHORT. European Neuropsychopharmacology, 29, S1011.

Ormel, J., Hartman, C. A., \& Snieder, H. (2019). The genetics of depression: successful genomewide association studies introduce new challenges. Translational psychiatry, 9(1), 1-10.

Parra-Guillen, Z. P., Fontanellas, A., Jiang, L., Jericó, D., Martini, P., Vera-Yunca, D., . . . Troconiz, I. F. (2020). Disease pharmacokinetic-pharmacodynamic modelling in acute intermittent porphyria to support the development of mRNA-based therapies. British Journal of Pharmacology.

Pastor, P. D. H., Du, X., Fazal, S., Davies, A. N., \& Gomez, C. M. (2018). Targeting the CACNA1A IRES as a treatment for spinocerebellar ataxia type 6. The Cerebellum, 17(1), 72-77.

Patterson, M. C., Mengel, E., Vanier, M. T., Moneuse, P., Rosenberg, D., \& Pineda, M. (2020). Treatment outcomes following continuous miglustat therapy in patients with NiemannPick disease Type C: a final report of the NPC Registry. Orphanet journal of rare diseases, 15, 1-10.

Peng, A., Cai, J., Lai, W., Zhang, L., Qiu, X., Zhu, X., . . Chen, L. (2018). Autoimmune Aquaporin-4 Channelopathy presented with psychiatric symptoms: a case report.

Pischik, E., \& Kauppinen, R. (2015). An update of clinical management of acute intermittent porphyria. The application of clinical genetics, 8, 201.

Pizzi, M. A., Alejos, D., Hasan, T. F., Atwal, P. S., Krishnaiengar, S. R., \& Freeman, W. D. (2019). Adult presentation of ornithine transcarbamylase deficiency: 2 illustrative cases of phenotypic variability and literature review. The Neurohospitalist, 9(1), 30-36.

Platt, D., \& Griggs, R. C. (2012). Use of acetazolamide in sulfonamide-allergic patients with neurologic channelopathies. Archives of neurology, 69(4), 527-529. 
Pokora, P., Jezela-Stanek, A., Różdżyńska-Świątkowska, A., Jurkiewicz, E., Bogdańska, A., Szymańska, E., .. . Stawiński, P. (2019). Mild phenotype of glutaric aciduria type 1 in polish patients-novel data from a group of 13 cases. Metabolic brain disease, 34(2), 641649.

Prasad, C., Rupar, T., \& Prasad, A. N. (2011). Pyruvate dehydrogenase deficiency and epilepsy. Brain and Development, 33(10), 856-865.

Pridmore, C. L., Clarke, J. T., \& Blaser, S. (1995). Ornithine transcarbamylase deficiency in females: an often overlooked cause of treatable encephalopathy. Journal of child neurology, 10(5), 369-374.

Purty, A., Nestadt, G., Samuels, J. F., \& Viswanath, B. (2019). Genetics of obsessive-compulsive disorder. Indian journal of psychiatry, 61(Suppl 1), S37.

Ramsay, J., Morton, J., Norris, M., \& Kanungo, S. (2018). Organic acid disorders. Annals of translational medicine, 6(24).

Ratna, N., Kamble, N. L., Venkatesh, S. D., Purushottam, M., Pal, P. K., \& Jain, S. (2020). Psychiatric morbidity and poor follow-up underlie suboptimal functional and survival outcomes in Huntington's disease. BMC neurology, 20(1), 1-6.

Rees, E., Walters, J. T., Chambert, K. D., O'Dushlaine, C., Szatkiewicz, J., Richards, A. L., . . . Moran, J. L. (2014). CNV analysis in a large schizophrenia sample implicates deletions at 16p12. 1 and SLC1A1 and duplications at 1p36. 33 and CGNL1. Hum Mol Genet, 23(6), 1669-1676.

Richards, S., Aziz, N., Bale, S., Bick, D., Das, S., Gastier-Foster, J., . . Spector, E. (2015). Standards and guidelines for the interpretation of sequence variants: a joint consensus recommendation of the American College of Medical Genetics and Genomics and the Association for Molecular Pathology. Genetics in Medicine, 17(5), 405-423.

S Grewal, A., Beniwal, M., Pandita, D., S Sekhon, B., \& Lather, V. (2016). Recent Updates on peroxisome proliferator-activated receptor $\delta$ agonists for the treatment of metabolic syndrome. Medicinal Chemistry, 12(1), 3-21.

Saitoh, A., Makino, Y., Hashimoto, T., Yamada, M., Gotoh, L., Sugiyama, A., . . Yamada, M. (2015). The voltage-gated sodium channel activator veratrine induces anxiogenic-like behaviors in rats. Behavioural brain research, 292, 316-322.

Sanches, M., Quevedo, J., \& Soares, J. C. (2019). Treatment Resistance in Bipolar Disorders Treatment Resistance in Psychiatry (pp. 139-150): Springer.

Sanju, S., Tullu, M. S., Seshadri, N., \& Agrawal, M. (2020). Glutaric Aciduria Type 1: A Case Report and Review of Literature. Journal of Pediatric Intensive Care. 
Santoro, L., Manganelli, F., Fortunato, M. R., Soldovieri, M. V., Ambrosino, P., Iodice, R., . . . Taglialatela, M. (2011). A new Italian FHM2 family: clinical aspects and functional analysis of the disease-associated mutation. Cephalalgia, 31(7), 808-819.

Satogami, K., Takahashi, S., Kose, A., \& Shinosaki, K. (2017). Schizophrenia-like symptoms in a patient with Leigh syndrome. Asian journal of psychiatry, 25, 249-250.

Saudubray, J.-M., \& Garcia-Cazorla, À. (2018). Inborn errors of metabolism overview: pathophysiology, manifestations, evaluation, and management. Pediatric Clinics, 65(2), 179-208.

Saudubray, J.-M., \& Sedel, F. (2009). Inborn errors of metabolism in adults. Ann Endocrinol (Paris), 70, 14-24.

Schirinzi, T., Vasco, G., Aiello, C., Rizzo, C., Sancesario, A., Romano, A., . . Castelli, E. (2019). Natural history of a cohort of ABCD 1 variant female carriers. European Journal of Neurology, 26(2), 326-332.

Schmunk, G., \& Gargus, J. J. (2013). Channelopathy pathogenesis in autism spectrum disorders. Frontiers in genetics, 4, 222.

Sedel, F., Baumann, N., Turpin, J.-C., Lyon-Caen, O., Saudubray, J.-M., \& Cohen, D. (2007). Psychiatric manifestations revealing inborn errors of metabolism in adolescents and adults. Journal of inherited metabolic disease, 30(5), 631-641.

Shamim, D., \& Alleyne, K. (2017). X-linked adult-onset adrenoleukodystrophy: Psychiatric and neurological manifestations. SAGE open medical case reports, 5, 2050313 X17741009.

Silva, S. E., Cristina, A., Rocha, N. P., Miranda, A. S., \& Teixeira, A. L. (2019).

Neuropsychiatric disorders in chronic kidney disease. Frontiers in pharmacology, 10, 932.

Simons, A., Eyskens, F., Glazemakers, I., \& van West, D. (2017). Can psychiatric childhood disorders be due to inborn errors of metabolism? European child \& adolescent psychiatry, 26(2), 143-154.

Sriretnakumar, V., Harripaul, R., Vincent, J. B., Kennedy, J. L., \& So, J. (2019). Enrichment of pathogenic variants in genes associated with inborn errors of metabolism in psychiatric populations. American Journal of Medical Genetics Part B: Neuropsychiatric Genetics, 180(1), 46-54. doi: 10.1002/ajmg.b.32702

Sriretnakumar, V., Zai, C. C., Wasim, S., Barsanti-Innes, B., Kennedy, J. L., \& So, J. (2019). Copy number variant syndromes are frequent in schizophrenia: progressing towards a CNV-schizophrenia model. Schizophrenia research.

Stenson, P. D., Mort, M., Ball, E. V., Evans, K., Hayden, M., Heywood, S., . . Cooper, D. N. (2017). The Human Gene Mutation Database: towards a comprehensive repository of 
inherited mutation data for medical research, genetic diagnosis and next-generation sequencing studies. Human Genetics, 1-13.

Stepien, K. M., Geberhiwot, T., Hendriksz, C. J., \& Treacy, E. P. (2019). Challenges in diagnosing and managing adult patients with urea cycle disorders. Journal of inherited metabolic disease, 42(6), 1136-1146.

Suddaby, J. S., Silver, J., \& So, J. (2019). Understanding the schizophrenia phenotype in the first patient with the full SCN2A phenotypic spectrum. Psychiatr Genet, 29(3), 91-94.

Tansey, K. E., Rees, E., Linden, D., Ripke, S., Chambert, K., Moran, J., . . Walters, J. (2016). Common alleles contribute to schizophrenia in CNV carriers. Molecular psychiatry, 21(8), 1085-1089.

Tarnacka, B., Szeszkowski, W., Buettner, J., Gołębiowski, M., Gromadzka, G., \& Członkowska, A. (2009). Heterozygous carriers for Wilson's disease-magnetic spectroscopy changes in the brain. Metabolic brain disease, 24(3), 463-468.

Team, R. C. (2017). R: A language and environment for statistical computing. Vienna, Austria. Retrieved from https://www.R-project.org/

Tishler, P. V., Woodward, B., O'Connor, J., Holbrook, D. A., Seidman, L. J., Hallett, M., \& Knighton, D. J. (1985). High prevalence of intermittent acute porphyria in a psychiatric patient population. Am J Psychiatry.

Trakadis, Y. J., Fulginiti, V., \& Walterfang, M. (2018). Inborn errors of metabolism associated with psychosis: literature review and case-control study using exome data from 5090 adult individuals. Journal of inherited metabolic disease, 41(4), 613-621.

Vigo, D., Thornicroft, G., \& Atun, R. (2016). Estimating the true global burden of mental illness. The Lancet Psychiatry, 3(2), 171-178.

Walterfang, M., Bonnot, O., Mocellin, R., \& Velakoulis, D. (2013). The neuropsychiatry of inborn errors of metabolism. J Inherit Metab Dis, 36(4), 687-702.

Waters, D., Adeloye, D., Woolham, D., Wastnedge, E., Patel, S., \& Rudan, I. (2018). Global birth prevalence and mortality from inborn errors of metabolism: a systematic analysis of the evidence. Journal of global health, 8(2).

Weiss, P. H., \& Fink, G. R. (2009). Grapheme-colour synaesthetes show increased grey matter volumes of parietal and fusiform cortex. Brain, 132(1), 65-70.

WHO. (2020). Genes and human diseases. Retrieved 29/07/2020, 2020, from https://www.who.int/genomics/public/geneticdiseases/en/index2.html

Wiesinger, C., Eichler, F. S., \& Berger, J. (2015). The genetic landscape of X-linked adrenoleukodystrophy: inheritance, mutations, modifier genes, and diagnosis. The application of clinical genetics, 8, 109. 
Winquist, R. J., \& Cohen, C. J. (2018). Integration of biological/pathophysiological contexts to help clarify genotype-phenotype mismatches in monogenetic diseases. Childhood epilepsies associated with SCN2A as a case study. Biochemical Pharmacology, 151, 252262.

Wolff, M., Brunklaus, A., \& Zuberi, S. M. (2019). Phenotypic spectrum and genetics of SCN 2A-related disorders, treatment options, and outcomes in epilepsy and beyond. Epilepsia, 60, S59-S67.

Wolff, M., Johannesen, K. M., Hedrich, U. B., Masnada, S., Rubboli, G., Gardella, E., .. . Villard, L. (2017). Genetic and phenotypic heterogeneity suggest therapeutic implications in SCN2A-related disorders. Brain, 140(5), 1316-1336.

Wu, B., Wang, Y.-Z., Cao, L.-R., Zhang, R.-P., Fang, Y.-L., Zhang, Y.-Q., \& Cai, C.-Q. (2018). Case Report A novel mutation in ornithine transcarbamylase gene identified from a Chinese child with ornithine transcarbamylase deficiency. Int J Clin Exp Med, 11(6), 6344-6350.

Wu, Y.-F. (2017). Recurrent hyperammonemia associated with olanzapine. Journal of clinical psychopharmacology, 37(3), 366-367.

Wykes, R., \& Lignani, G. (2018). Gene therapy and editing: Novel potential treatments for neuronal channelopathies. Neuropharmacology, 132, 108-117.

Yamakawa, K. (2016). Mutations of voltage-gated sodium channel genes SCN1A and SCN2A in epilepsy, intellectual disability, and autism Neuronal and synaptic dysfunction in autism Spectrum disorder and intellectual disability (pp. 233-251): Elsevier.

Yousefichaijan, P., Salehi, B., Rafiei, M., Taherahmadi, H., Sharafkhah, M., \& Naziri, M. (2014). Prevalence of obsessive-compulsive disorder in pediatric and adolescent patients with chronic kidney disease. Journal of Pediatric Nephrology, 2(3), 107-109.

Yousefichaijan, P., Sharafkhah, M., Rafeie, M., \& Salehi, B. (2016). Obsessive compulsive inventory-child version (OCV-CI) to evaluate obsessive compulsive disorder in children with early stages of chronic kidney disease: a case control study. Nephro-Urology Monthly, 8(1).

Zaman, T., Helbig, K. L., Clatot, J., Thompson, C. H., Kang, S. K., Stouffs, K., . . Parrini, E. (2020). SCN3A-related neurodevelopmental disorder: A spectrum of epilepsy and brain malformation. Annals of neurology. 